\section{Y-12}
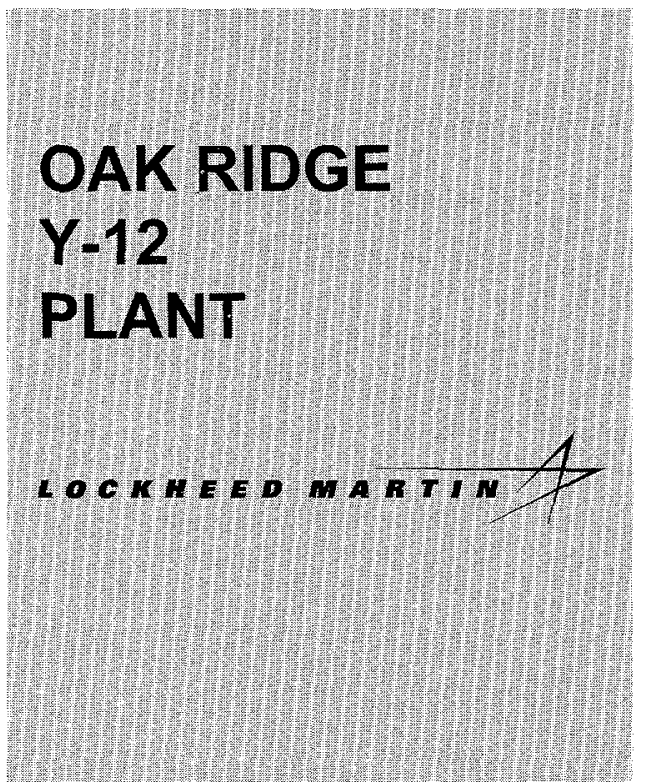

(1)
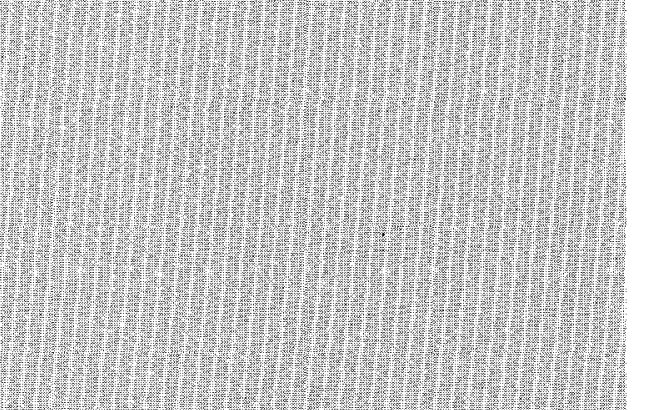

1.t.

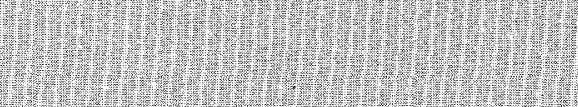

(1)

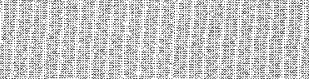

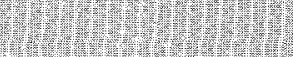

(1)
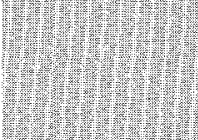

\section{EMERGENCY PLANNING AND COMMUNITY}

RIGHT-TO-KNOW ACT

\section{SECTION 312 TIER TWO REPORT FORMS}

\author{
R. A. Evans \\ Environmental Compliance Department \\ Environment, Safety, Health and Quality Organization
}

Prepared by the Oak Ridge Y-12 Plant

Oak Ridge, Tennessee 37831 managed by

Lockheed Martin Energy Systems, Inc. for the U.S. Department of Energy under Contract DE-AC05-84OR21400 


\section{DISCLAIMER}

This report was prepared as an account of work sponsored by an agency of the United States Government. Neither the United States Government nor any agency thereof, nor any of their employees, makes any warranty, express or implied, or assumes any legal liability or responsibility for the accuracy, completeness, or usefulness of any information, apparatus, product, or process disclosed, or represents that its use would not infringe privately owned rights. Reference herein to any specific commercial product, pracess, or service by trade name, trademark, manufacturer, or otherwise, does not necessarily constitute or imply its endorsement, recommendation, or favoring by the United States Government or any agency thereof. The views and opinions of authors expressed herein do not necessarily state or reflect those of the United States Government or any agency thereof. 


\section{DISCLAIMER}

Portions of this document may be illegible in electronic image products. Images are produced from the best available original document. 


\title{
EMERGENCY PLANNING AND COMMUNITY RIGHT-TO-KNOW ACT SECTION 312 TIER TWO REPORT FORMS
}

\author{
R. A. Evans \\ Environmental Compliance Department \\ Environment, Safety, Health and Quality Organization
}

February 2000

Prepared by the

Oak Ridge Y-12 Plant

Oak Ridge, Tennessee 37831

managed by

Lockheed Martin Energy Systems, Inc.

for the U.S. Department of Energy

under Contract DE-AC05-84OR21400 


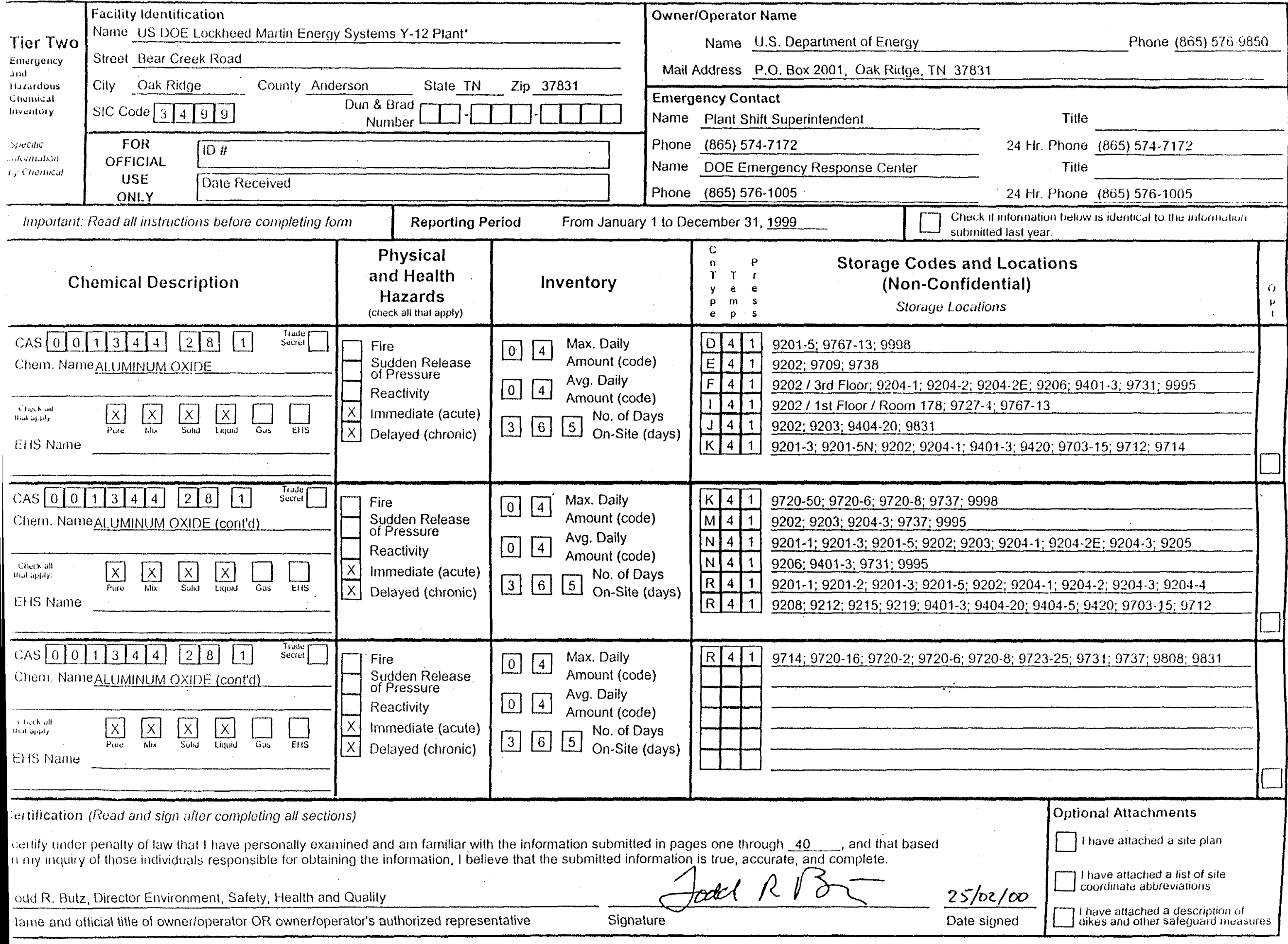




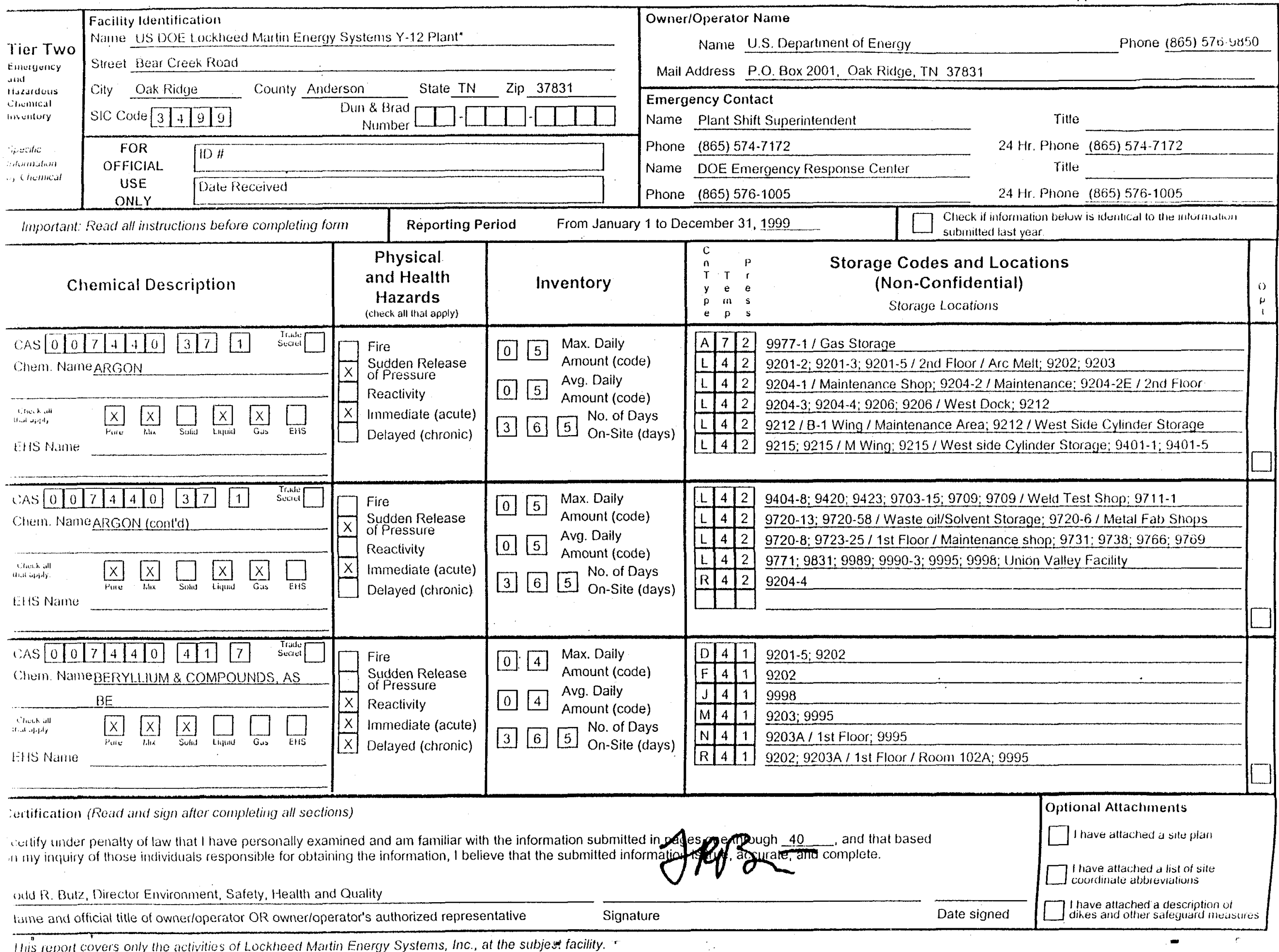




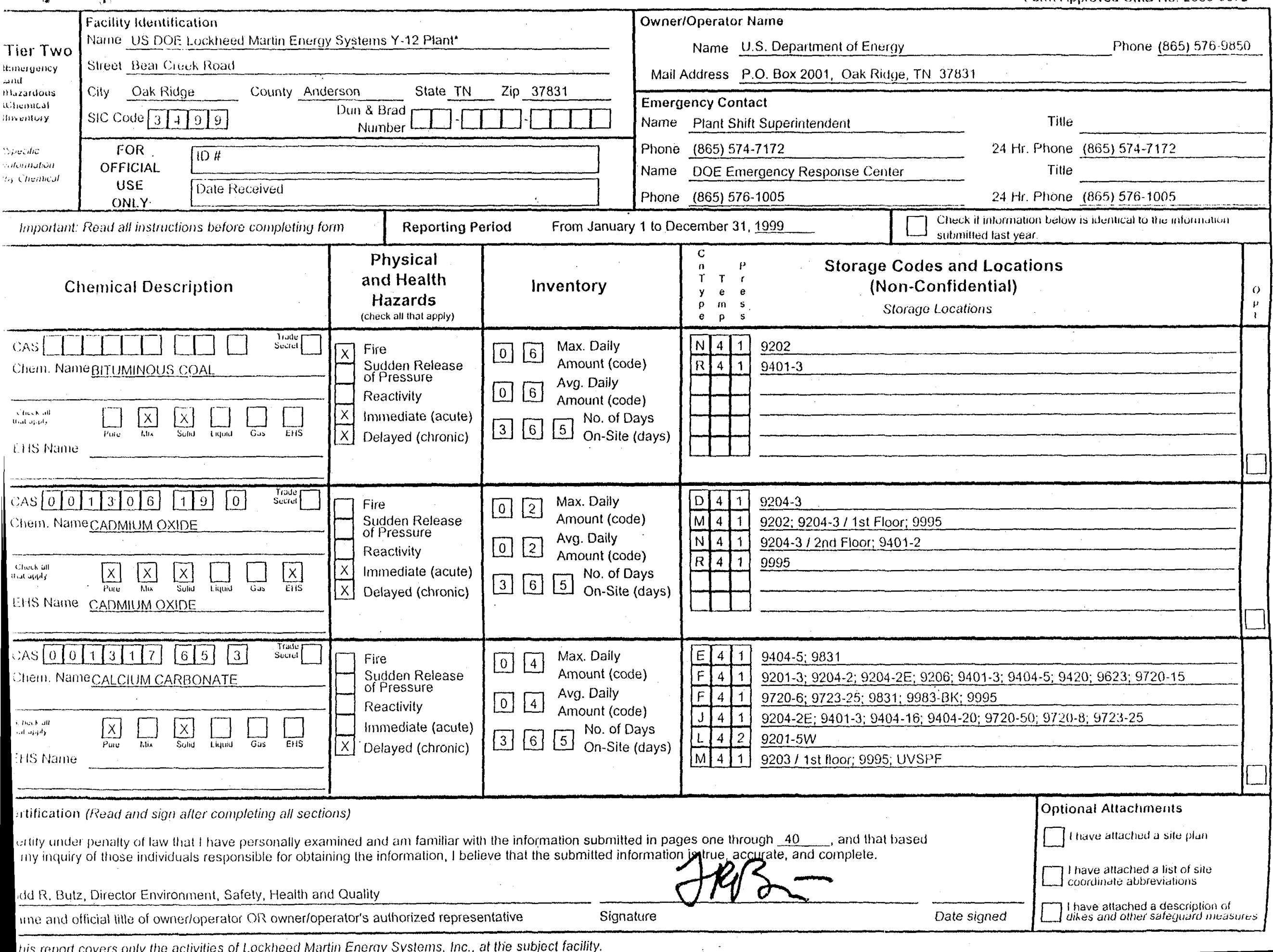




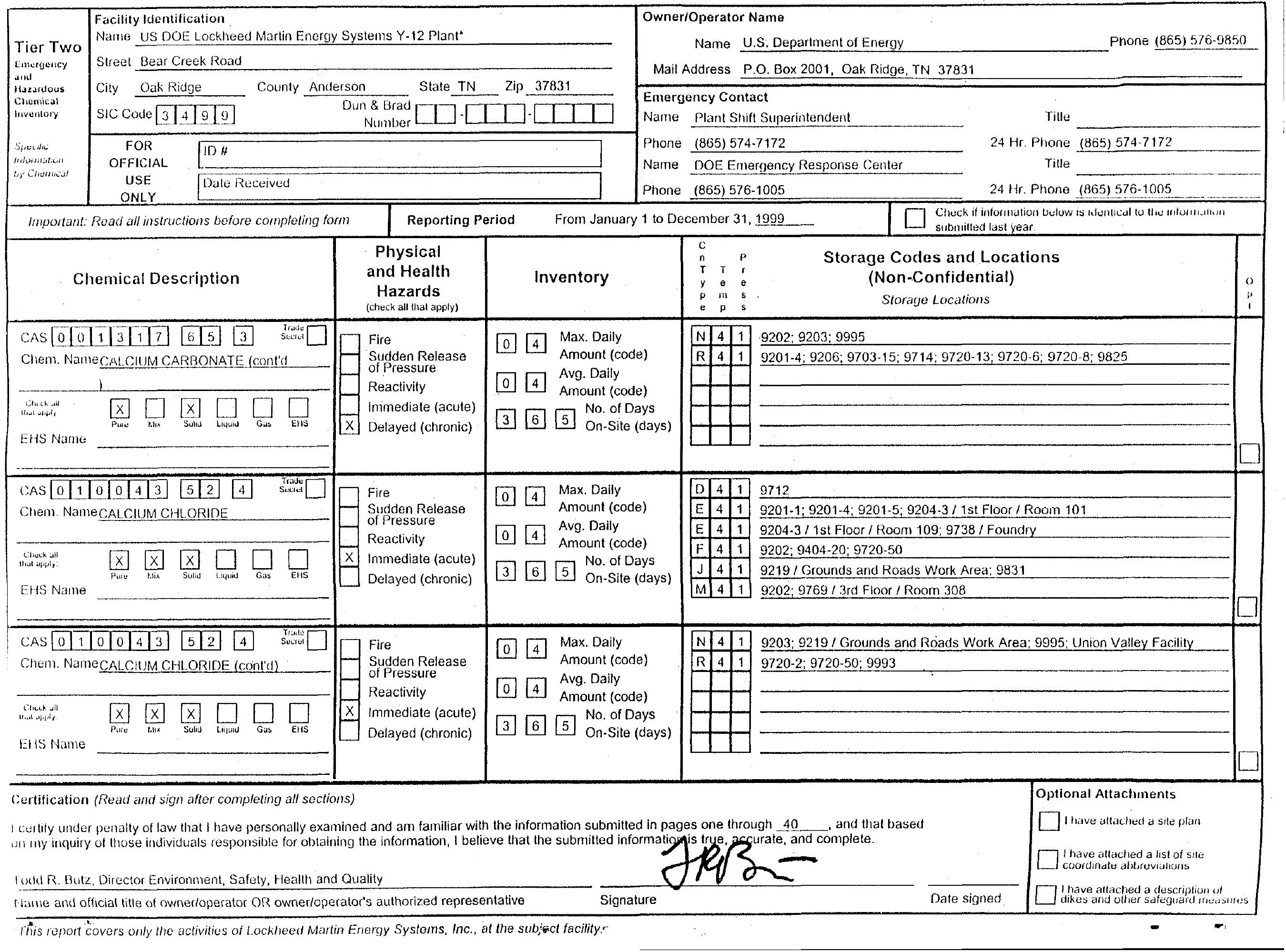




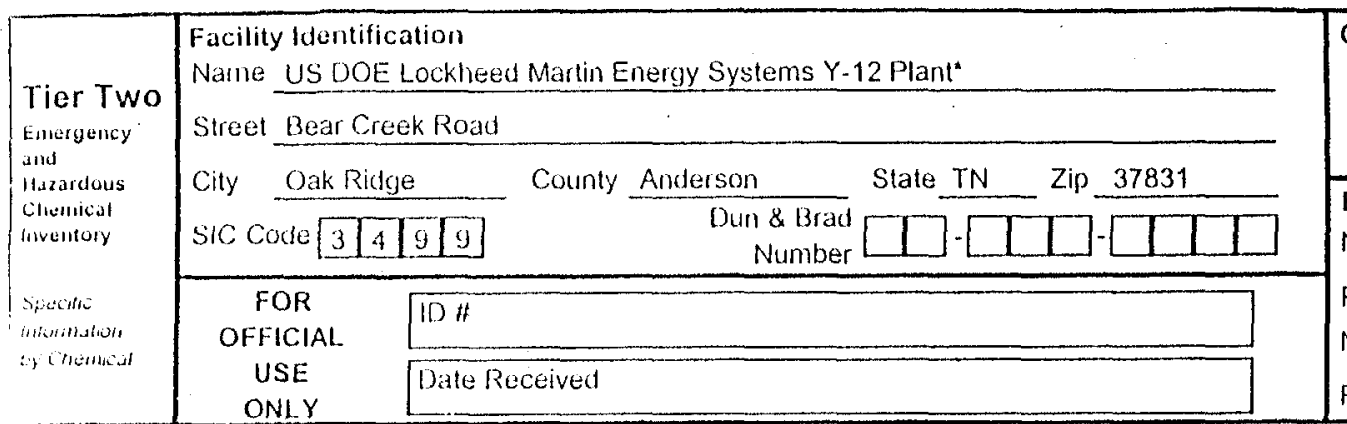

Owner/Operator Name

Name U.S. Department of Energy Phone (865) 576.9850 Mail Address P.O. Box 2001, Oak Ridge, TN 37831

\section{Emergency Contact}

Name Plant Shift Superintendent

Phone (865) 574-7172

Name DOE Emergency Response Center

Phone (865) 576-1005

Important: Read all instructions before completing form Reporting Period

From January 1 to December 31, 1999

\section{Physical}

Chemical Description and Health Hazards (check all that apply)

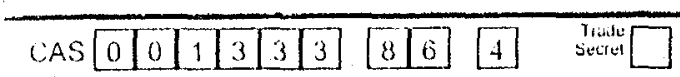
Chem. Name CARBON BLACK (cont'd)

\section{$x$ Fire}

Sudden Release of Pressure

Reactivity

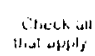

EHS Name

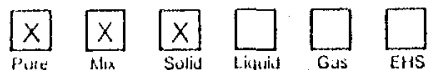

$X$ Immediate (acute)

Delayed (chronic)

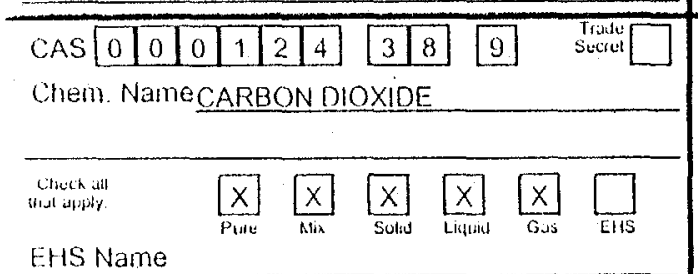

\section{$\square$ Fire}

$\mathrm{X}$ Sudden Release

Reactivity

$X$ Immediate (acute)

Delayed (chronic)

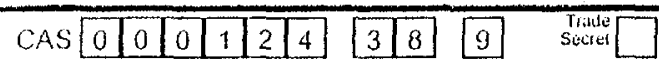
Chem. Name CARBON DIOXIDE (cont'd)

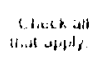

EHS Name

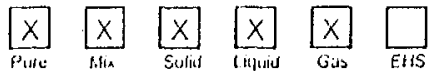

\section{$\square$ Fire}

$x$ Sudden Release

of Pressure

Reactivity

$x$ Immediate (acute)

Delayed (chronic)
Inventory

Amount (code)

Avg. Daily

Amount (code)

356 No. of Days

$\begin{array}{lll}C & & \\ n & & P \\ T & T & r \\ y & e & e \\ \rho & m & s \\ e & p & s\end{array}$

\begin{tabular}{|l|l|l|l|l|}
\hline$N$ & 4 & 1 \\
\hline & $9202 ; 9203 ; 9204-2 ; 9204-3 ; 9204-4 ; 9731 ; 9995$ \\
\hline
\end{tabular}

$\begin{array}{llll}R & 4 & 1 & 9201-1 ; 9201-2 ; 9201-5 ; 9202 ; 9204-1 ; 9204-2 ; 9208 ; 9212 ; 9215 ; 9420\end{array}$

$\begin{array}{llll}R & 4 & 1 & 9703-15 ; 9709 ; 9710-2 ; 9720-1 ; 9720-6 ; 9720-8 ; 9725 ; 9737 ; 9738 ; 9831\end{array}$ \begin{tabular}{l|l|l|l|l|l}
\hline$R$ & 4 & 1 & $9995 ;$ UVSPF \\
\hline
\end{tabular}
Storage Codes and Locations Non-Confidential)

Storage Locations
Title

4 Hr. Phone (865) 574-7172

Tille

$24 \mathrm{Hr}$. Phone (865) 576-1005

\section{Max. Daily} Amount (code)

0 Avg. Daily

Amount (code) 5 No. of Days 365 On-Site (days)

\section{Max. Daily}

4. Amount (code)

[ 4 Amount (code)

[3 6] 5 No. of Days
F[4]1 $9201-3 ; 9201-5 ; 9202 ; 9203 ; 9204-1 ; 9204-2 E ; 9204-4 ; 9206 ; 9212 ; 9714$

\begin{tabular}{lll}
\hline$F$ & 4 & 2 \\
\hline$F$ & $4201-3 ; 9201-5 ; 9202 ; 9204-1 ; 9204-2 ; 9204-2 E ; 9204-4 ; 9206 ; 9212$ \\
\hline 215
\end{tabular}

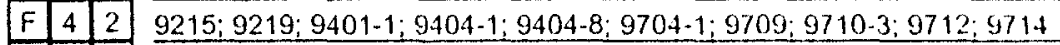

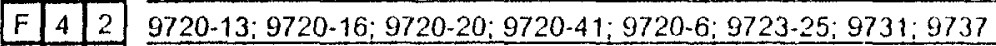

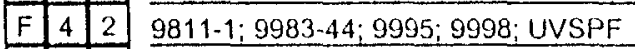

\begin{tabular}{|l|l|l|l|l|}
\hline L & 4 & 2 & $9204-2 E / 2 n d$ Floor; 9709; 9711-5; 9769; 9989; 9990-3; 9995 / BASEMENT \\
\hline
\end{tabular}

\section{\begin{tabular}{|l|l|l|}
\hline$L$ & 4 & 2 \\
\hline
\end{tabular}}

\begin{tabular}{|l|l|l|l|l|l|l}
\hline$L$ & 6 & 2 & 9401.1 \\
\hline$P$ & 4 & 2 & \\
\hline $9201-3.97$
\end{tabular}

\begin{tabular}{llll}
\hline$P$ & 4 & 2 & $9201-3 ; 9710-2 /$ Outside Tanker \\
\hline$P$ & 7 & 2 &
\end{tabular}

\begin{tabular}{lllll}
$P$ & 7 & 2 & $9710-2$ \\
\hline$R$ & 4 & 2 & $9201-1 ; 92$
\end{tabular}

\begin{tabular}{lll|l}
\hline$R$ & 4 & 2 & $9201-1 ; 9204-4 ; 9737 ; 9995$ \\
\hline$R$ & 6 & 2 & $9710-2$
\end{tabular} 

lupotant: Read all instructions before completing form

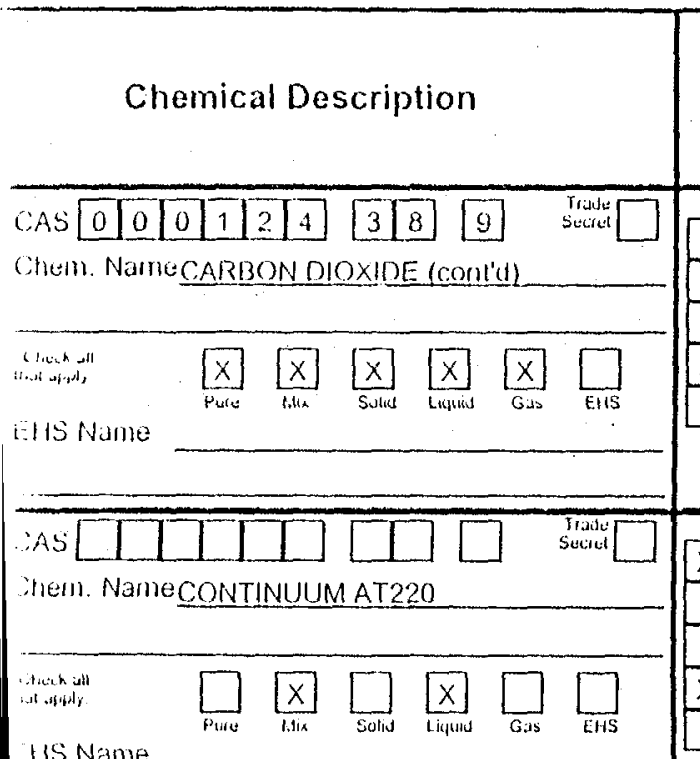

Reporting Period

From January 1 to December 31.1999.

Name U.S. Depariment of Energy

Mail Address P.O. Box 2001, Oak Ridge. TN 37831

\section{Emergency Contact}

Name Plant Shift Superintendent

Title

Phone (865) 574-7172

Name DOE Emergency Response Center

Phone (865) 576-1005

24 Hr. Phone (865) 574.7172

Title

$24 \mathrm{Hr}$. Phone (865) 576-1005

\section{Physical} and Health Hazards (check all that appiy)

\begin{tabular}{|c|c|}
\hline & Fire \\
\hline$x$ & $\begin{array}{l}\text { Sudden Release } \\
\text { of Pressure }\end{array}$ \\
\hline & Reactivity \\
\hline$x$ & Immediate (acute) \\
\hline & Delayed (chronic) \\
\hline
\end{tabular}

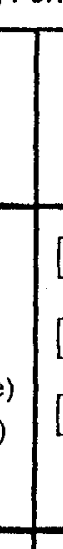

\begin{tabular}{|c|c|}
\hline$x$ & Fire \\
\hline & $\begin{array}{l}\text { Sudden Release } \\
\text { of Pressure }\end{array}$ \\
\hline & Reactivity \\
\hline$x$ & Immediate (acute) \\
\hline & Delayed (chronic) \\
\hline
\end{tabular}

$\begin{array}{lll}c & & \\ n & & p \\ r & T & r \\ y & e & e \\ p & m & s \\ e & \rho & s\end{array}$

Check if intomation Check if intormation $D$
submitted tast year.

\section{Storage Codes and Locations} (Non-Confidential)

Storage Locations

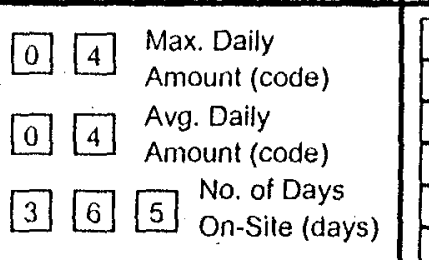

\begin{tabular}{|l|l|l|}
\hline$R$ & 7 & 1 \\
\hline$R$ & 7 & 2 \\
\hline & & \\
\hline & & \\
\hline & & \\
\hline & & \\
\hline
\end{tabular}

9995

$\frac{9995}{9710-2}$

00 Max. Daily Max. Daily

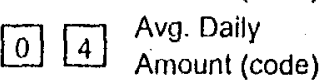

(3) 5 No. of Days

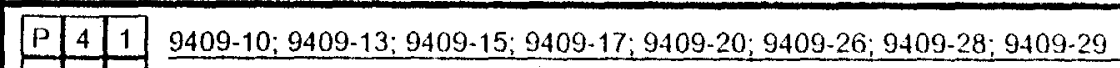
\begin{tabular}{|l|l|l|l|}
\hline$P$ & 4 & 1 \\
\hline$R$ & 4 & 1 & $9409-32 ; 9409-34 ; 9409-35$ \\
\hline
\end{tabular}

\begin{tabular}{|lll|l}
\hline$R$ & 4 & 1 & $9409-32$ \\
\hline$R$ & 4 & 1 \\
\hline
\end{tabular}

5 On-Site (days)

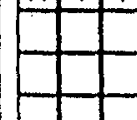

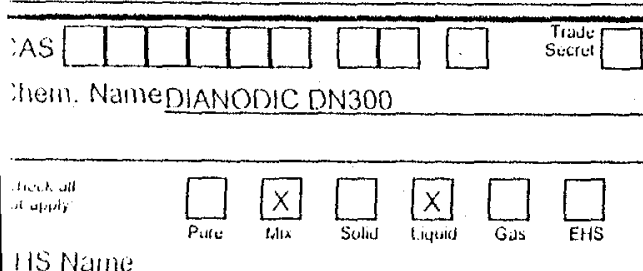

\section{$x$ Fire}

Sudden Release Pressure

Reactivity

$x$ Immediate (acute)

$\square$ Delayed (chronic)

\begin{tabular}{|c|c|c|}
\hline 0 & 4 & Max. Daily \\
\hline & 4 & Avg. Daily \\
\hline 10 & 4 & Amount (code) \\
\hline 3 & 6 & $5] \begin{array}{l}\text { No. of Days } \\
\text { On-Site (days) }\end{array}$ \\
\hline
\end{tabular}

\section{\begin{tabular}{|l|l|l|}
\hline$P$ & 4 & 1 \\
\hline$R$ & 4 & 1 \\
\hline
\end{tabular}

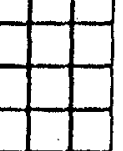

$9409-20 ; 9409-22 ; 9409-22 E ; 9409-23 ; 9409-73$

\section{\begin{tabular}{ll|l|l|l|l|l|l|l|}
\hline$R$ & 4 & 1 & $9409-22$ \\
\hline
\end{tabular}}

rification (Read and sign after completing all sections)

: thy under penalty of law that ? have personally examined and am famitiar with the Information submitted in pages one through 40 _.., and that based

iny inquiry of lhose individuals responsible for obtaining the information, I believe that the submitted informationjofrugacengate, and complete.

11 R. Butz, Director Environment, Safely, Health and Quatity

Yleizian-

illit and official title of ownerloperator OR owner/operator's authorized representative

Signature

Date signed

Optional Altachments

$\square$ inave atlached a silup plan $\square$ I have attached a list of site

is report covers only the activities of Lockheed Martin Energy Systems, Inc, at the subject facility. 


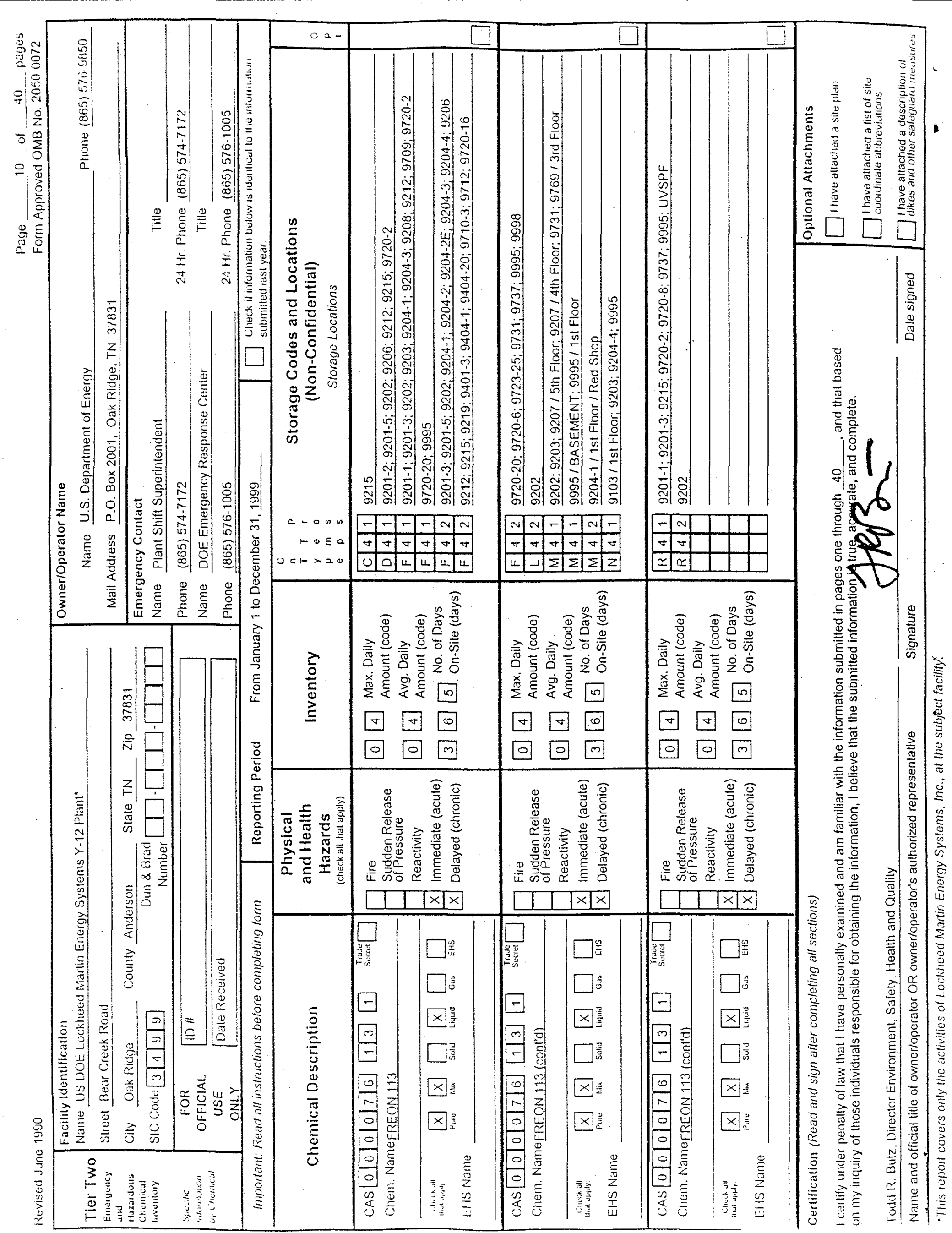




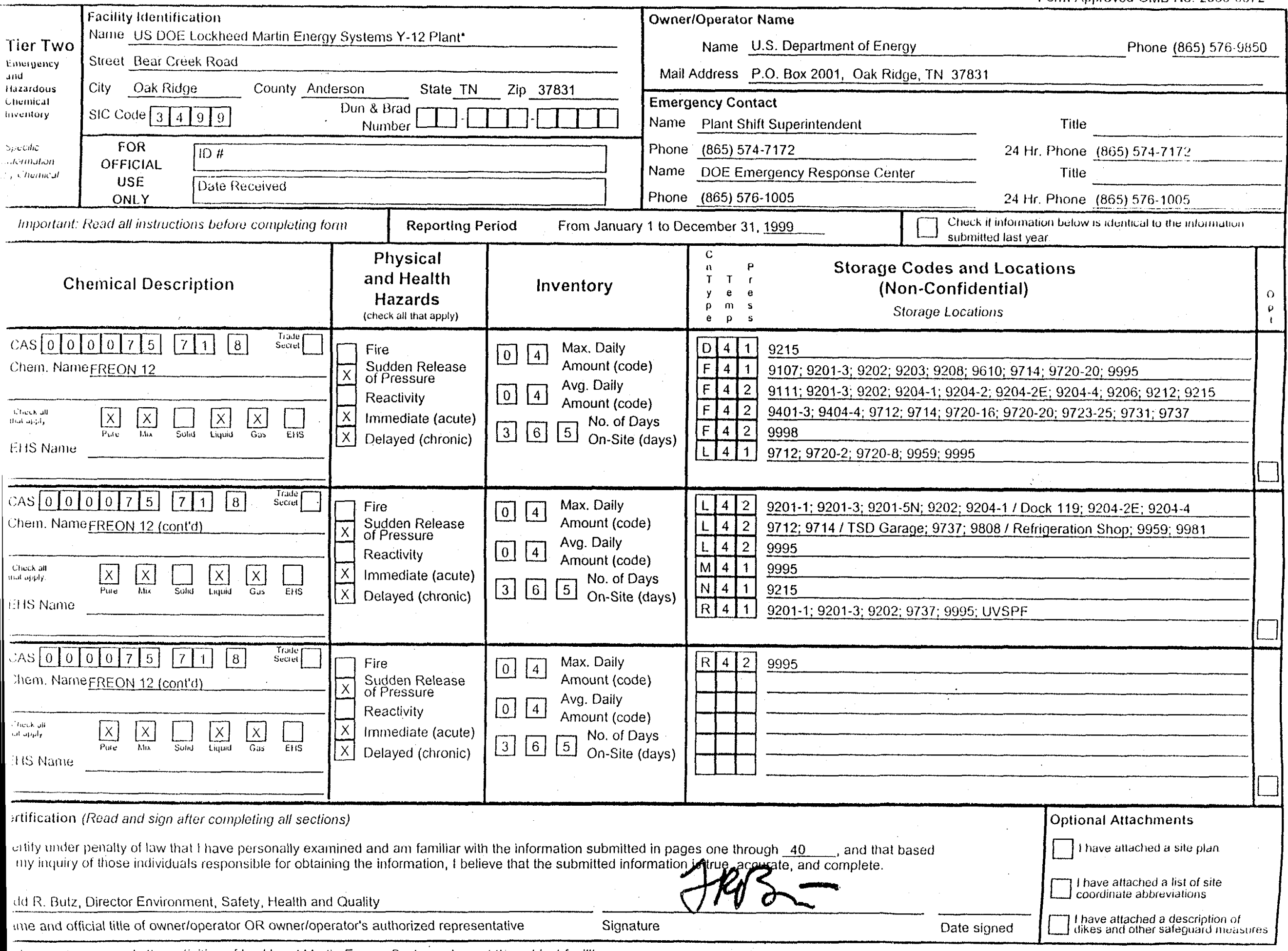

iis report covers only the aclivities of Lockheed Martin Energy Systems, Inc., at the subject facility. 


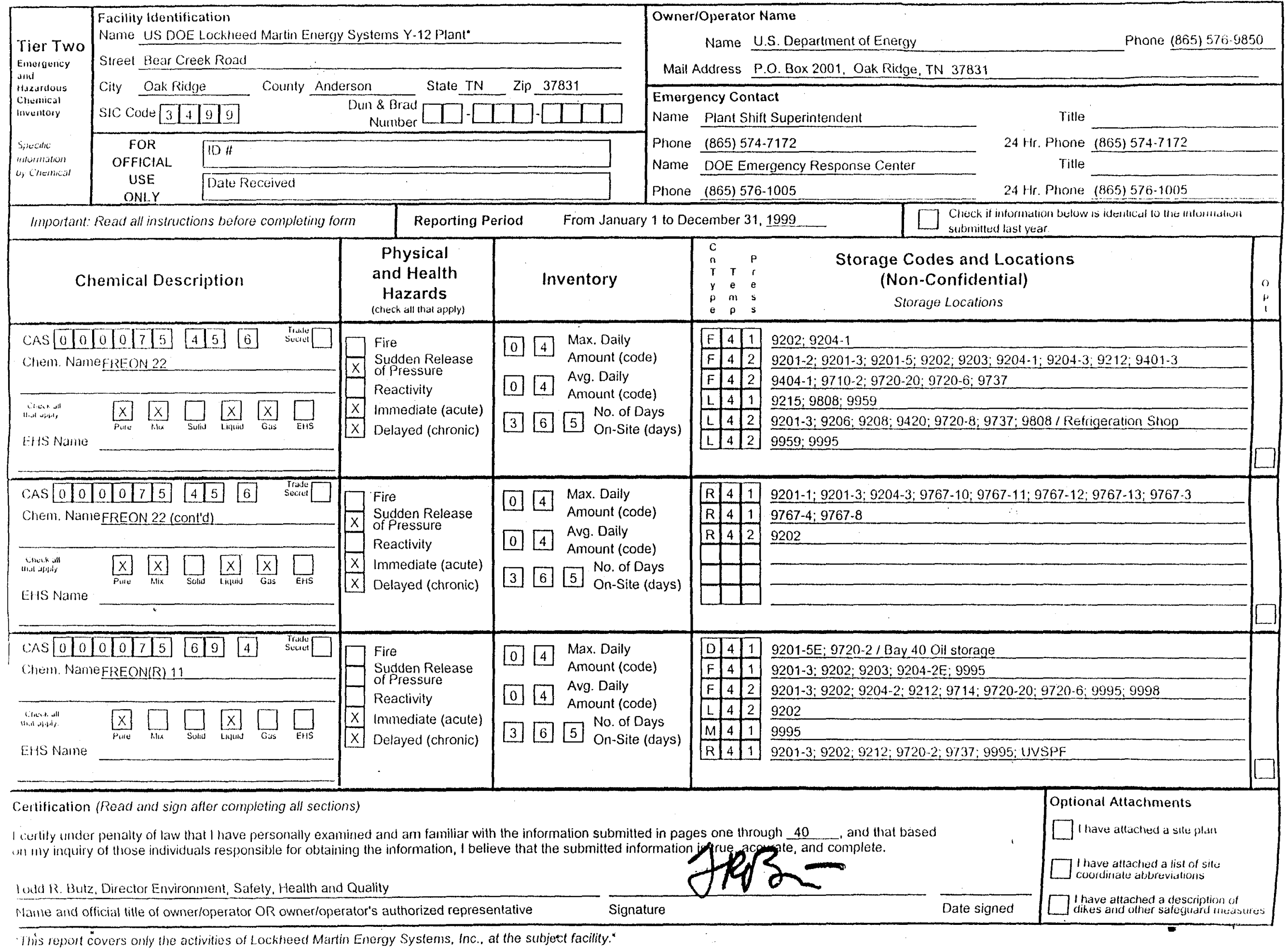


Facility ldentification

Tier Two

Emeryency

aind Chesmicial

tiventon

sidectic

misuritistion

b) Coretimisis

Name US DOE Lockheed Martin Energy Systems Y-12 Plant*

Street Bear Creck Road

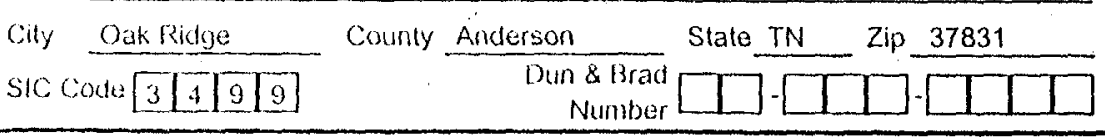

FOR
OFFICIAL
USE
ONLY

Name U.S. Department of Energy Phone (865) $5 \%$ r. 9850

Mail Address P.O. Box 2001, Oak Ridge, TN 37831

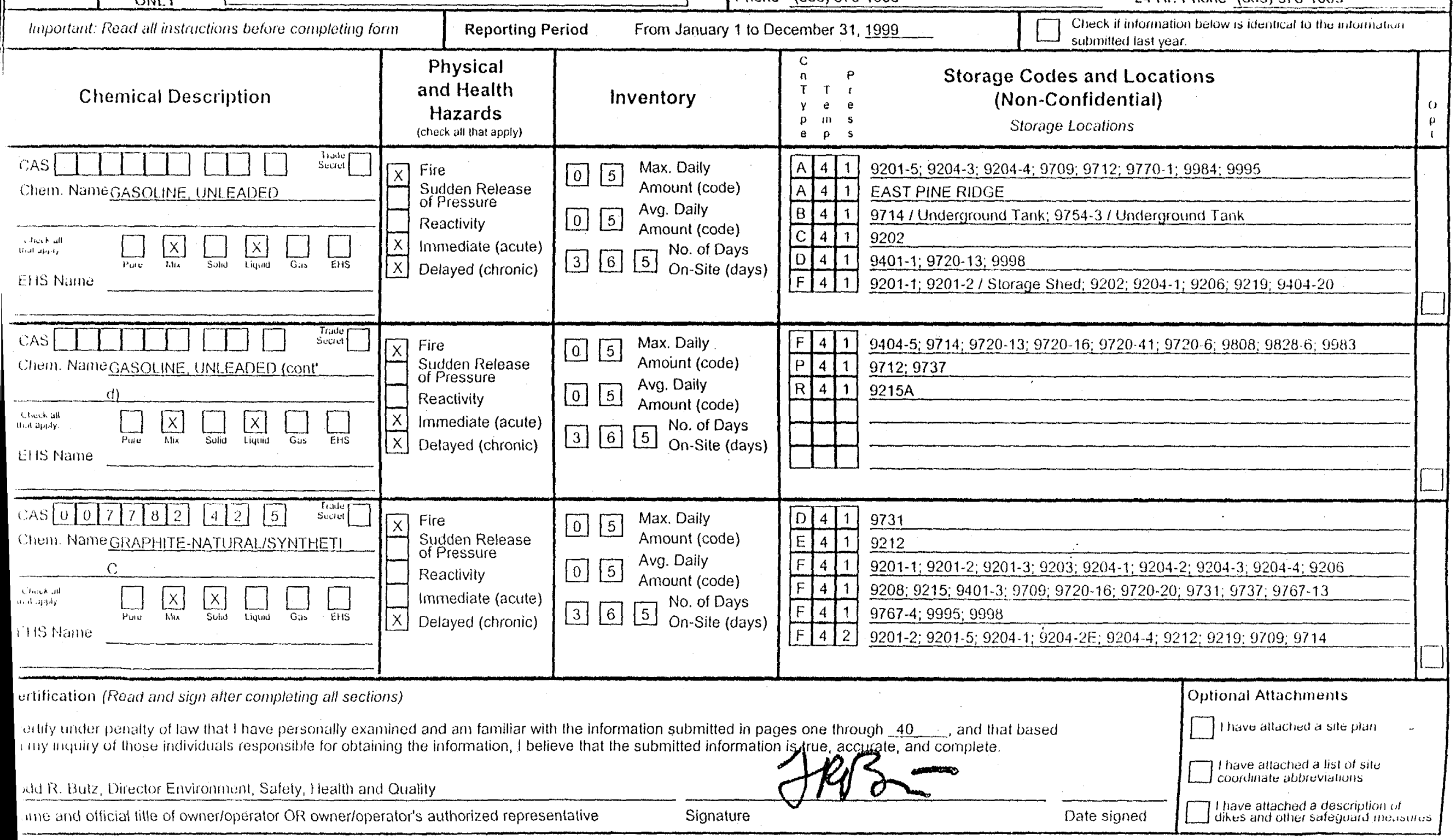

whe and officiat tille of owner/operator OR owner/operator's authorized representative Signature

\section{Emergency Contact}

Name Plant Shift Superintendent

Phone (865) $574-7172$

Name DOE Emergency Response Center

Phone (865) 576-1005

\begin{tabular}{|l|l|l|l|l|l}
\hline A & 4 & 1 & $9201-5 ; 9204-3 ; 9204-4 ; 9709 ; 9712 ; 9770-1 ; 9984 ; 9995$ \\
\hline$A$ & 4 & 1 & EASTPINE RID
\end{tabular}

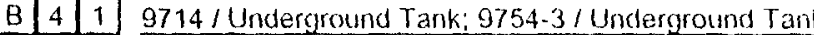

\begin{tabular}{llll}
\hline & 4 & 1 & 9202 \\
\hline & 4 & &
\end{tabular}

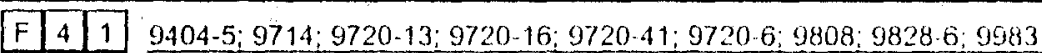

\begin{tabular}{llll}
$\mathrm{P}$ & 4 & 1 & $9712 ; 9737$ \\
\hline $\mathrm{R}$ & 4 & 1 & $9215 \mathrm{~A}$
\end{tabular}

\begin{tabular}{lllll}
$\mathrm{R}$ & 4 & 1 & $9215 \mathrm{~A}$ \\
\hline
\end{tabular}

5 Amount (code)

5 No. of Days

0 5ax. Daily

5 Avg Dally

(6) of Days

his ropont covers only the activitios of Lockined Martin Energy Systems, inc, at the subject facifity.
Tille

24 Hr. Plone (865) 574-7172

Title 


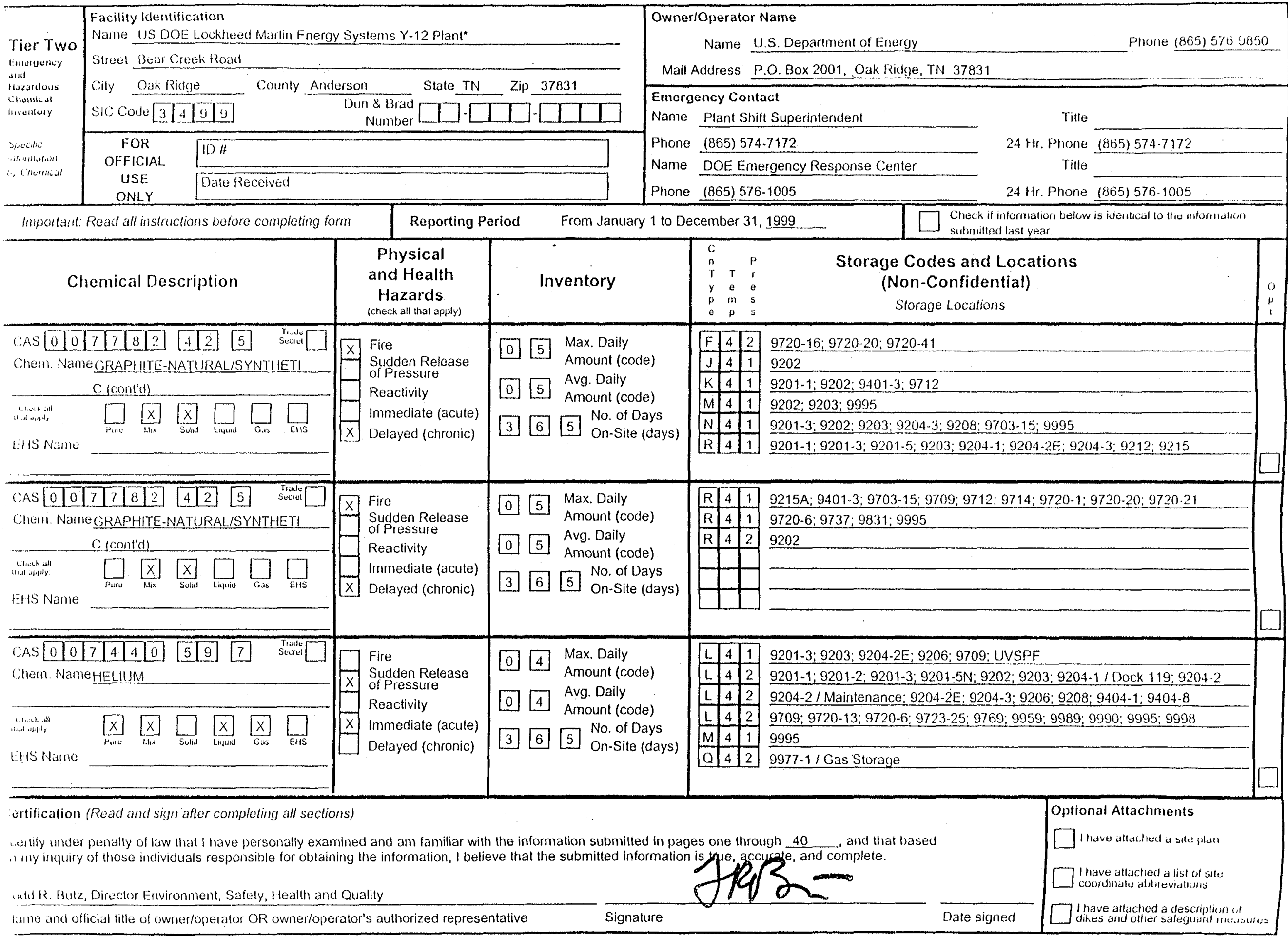

Ins repont covers only the activities of Lockheed Martin Energy Systems, Inc, at the subject facility. . 
Tier Two stimeryency miszardous hivinical iventiory

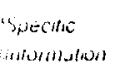
(i) chendid Facility ldentification

Name US DOE Lockheed Martin Energy Systems Y-12 Plant* Street Bear Creek Road

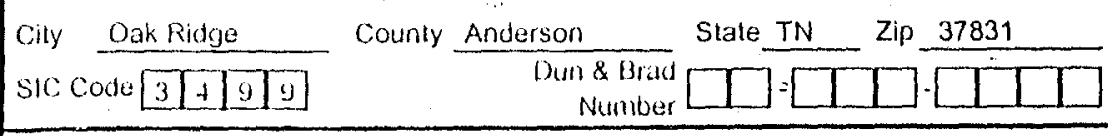

Name U.S. Department of Energy

Phone (865) 576.9850

Mail Address P.O. Box 2001, Oak Ringe, TN 37831

Emergency Contact

Name Plant Shift Superintemtent

Tille

FOR
OFFICIAL
USE
ONLY

Important: Read all instructions bofore completing form Reporting Period

From January 1 to December 31 , 1999

Phone (865) 576-1005

Phone (865) 574-7 172

Name DOE Emergency Response Center

to December 31, 1999
Chemical Description

Physical and Health Hazards (check all that apply)

$\operatorname{Cas[0[0]74]4[0]5]0]}$

Chem. Name HELIIIM (cont'd)

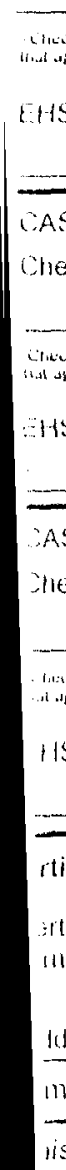
Fire $x$ of Pressure Reactivity $x$ Fire $x$ Sudden Re Reactivity intity

S Name

s[0]0/1/3/3/3 [7] [0]
Mem. Name HYOROGEN (cont'd)

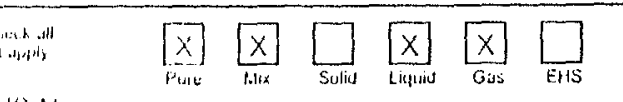

is Name

ification (Read and sign after completing all sections)

UR. Butz, Director Environment, Safety, Health and Quality Sudden Release

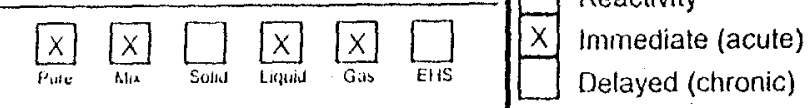

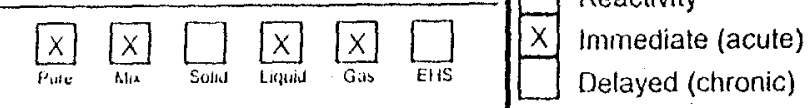

0
0
0
0
Inventory

20 Max. Daily [0] Amount (code) [3] 5 No. of Days 5 On-Site (days)

04 Max. Daily Amount (code) 0 Avg. Daily 4. Amount (code) (3) 6 No. of Days

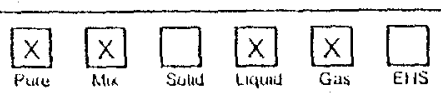
$x$ Inmediate (acute) $\square$ Delayed (chronic)

\section{$x$ Fire} $x$ Sudden Releas $x$ of Pressure Reactivily

$x$ Immediate (acute) Delayed (chronic)
[0] 4 Max. Daily
Amount (code)
[0] Avg. Daily
355 No. of Days

$24 \mathrm{Hr}$. Phone (865) 574-7172

Title

$24 \mathrm{Hr}$. Phone (865) 576-1005 tify under penally of law that I have personsally examined and am familiar with the information submitted in pages one through 40 _., and that based

wy inguicy of those individuals responsible for obtaining the information, I believe that the submitted information ignue accile, and complete.

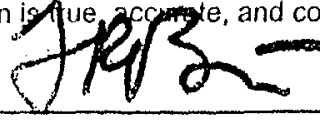

Storage Codes and Locations

(Non-Confidential)

Storage Locations

\begin{tabular}{|l|l|l|}
\hline$R$ & 4 & 1 \\
\hline$R$ & 4 & 2 \\
\hline$R$ & 7 & 2 \\
\hline & & \\
\hline & & \\
\hline & & \\
\hline
\end{tabular}

9215A; 9977-1: UVSPF

9204-4; 9995

9995

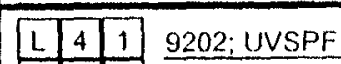

L 172 2 $2021-1 ; 9201-2 ; 9201-3 ; 9202 ; 9204-3 ; 9206 ; 9401-1 ; 9989 ; 9990 ; 9995$ \begin{tabular}{llll}
\hline L & 7 & 2 & 9995 \\
\hline$M$ & 4 & 1 & 9995
\end{tabular}

\begin{tabular}{llll}
$M$ & 4 & 1 & 9995 \\
\hline$N$ & 4 & 1 & 9202
\end{tabular}

\begin{tabular}{lllll}
\hline$N$ & 4 & 1 & 9202 \\
\hline & 4 & & \\
\hline 2013 &
\end{tabular}

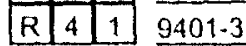

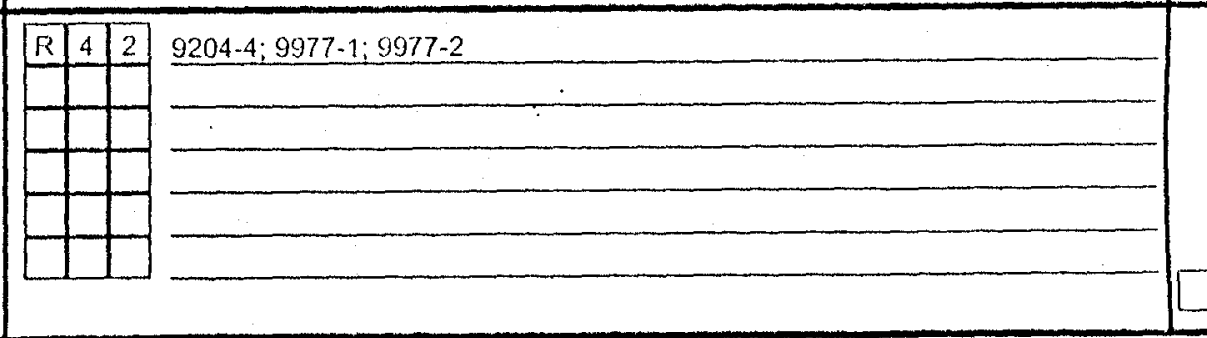

Optional Attachments

D hrave allached a site plun

$\square$ thave attached a list of site coordinate abbreviations

I have altached a dascription of
dikes and other safeguard nutedsures

he and official title of owner/operator OR owner/operator's authorized representative

Signature

is report covers only the activities of Lockheed Martin Energy Systems, Inc., at the subject facility. 


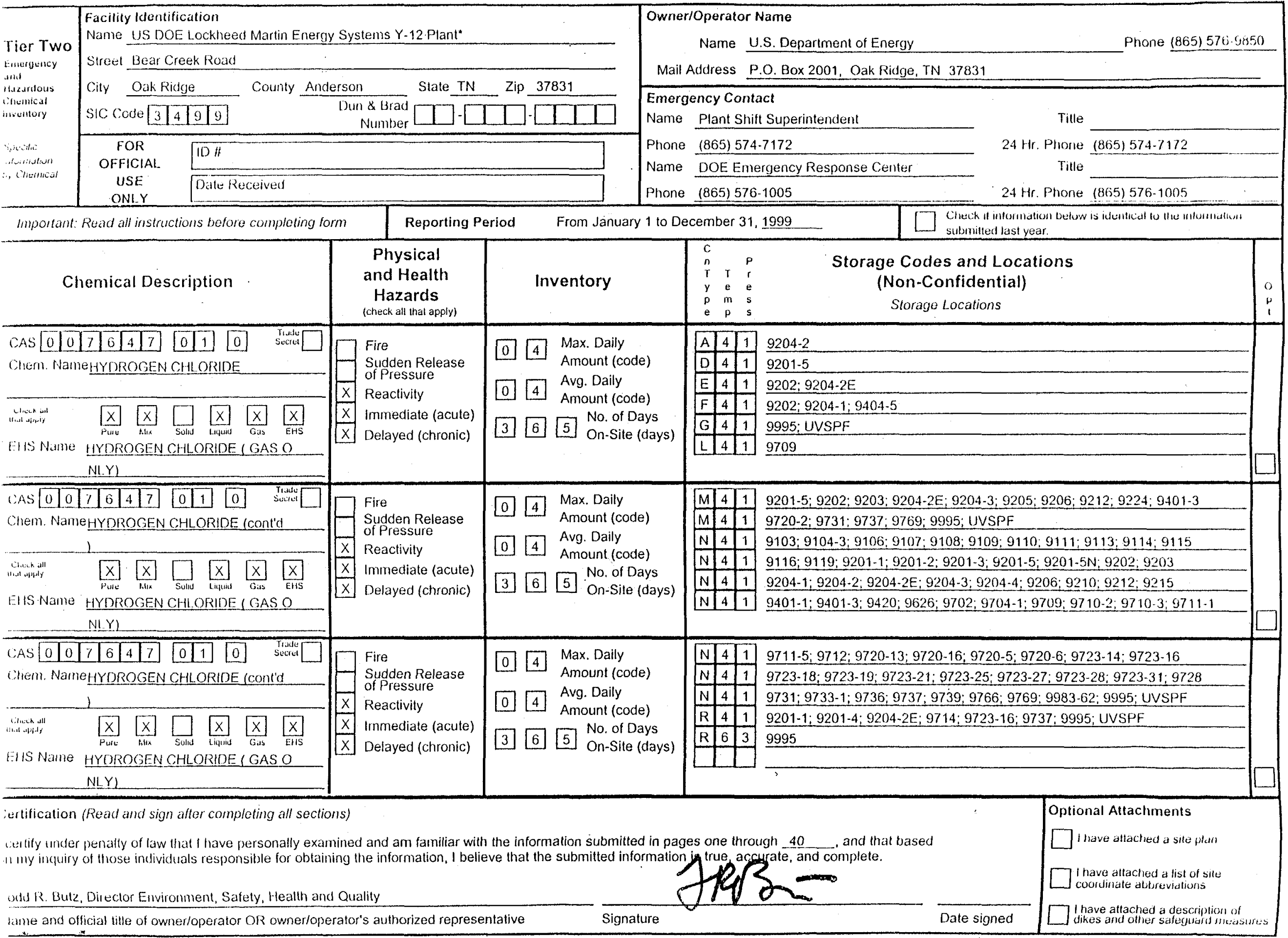

hame and official tille of owner/operator OR owner/operator's authorized representative Signature Date signed

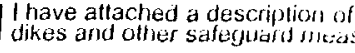
This leport covers only the activitios of Lochiced Matin Energy Systems, Inc, at the subject facility. " 


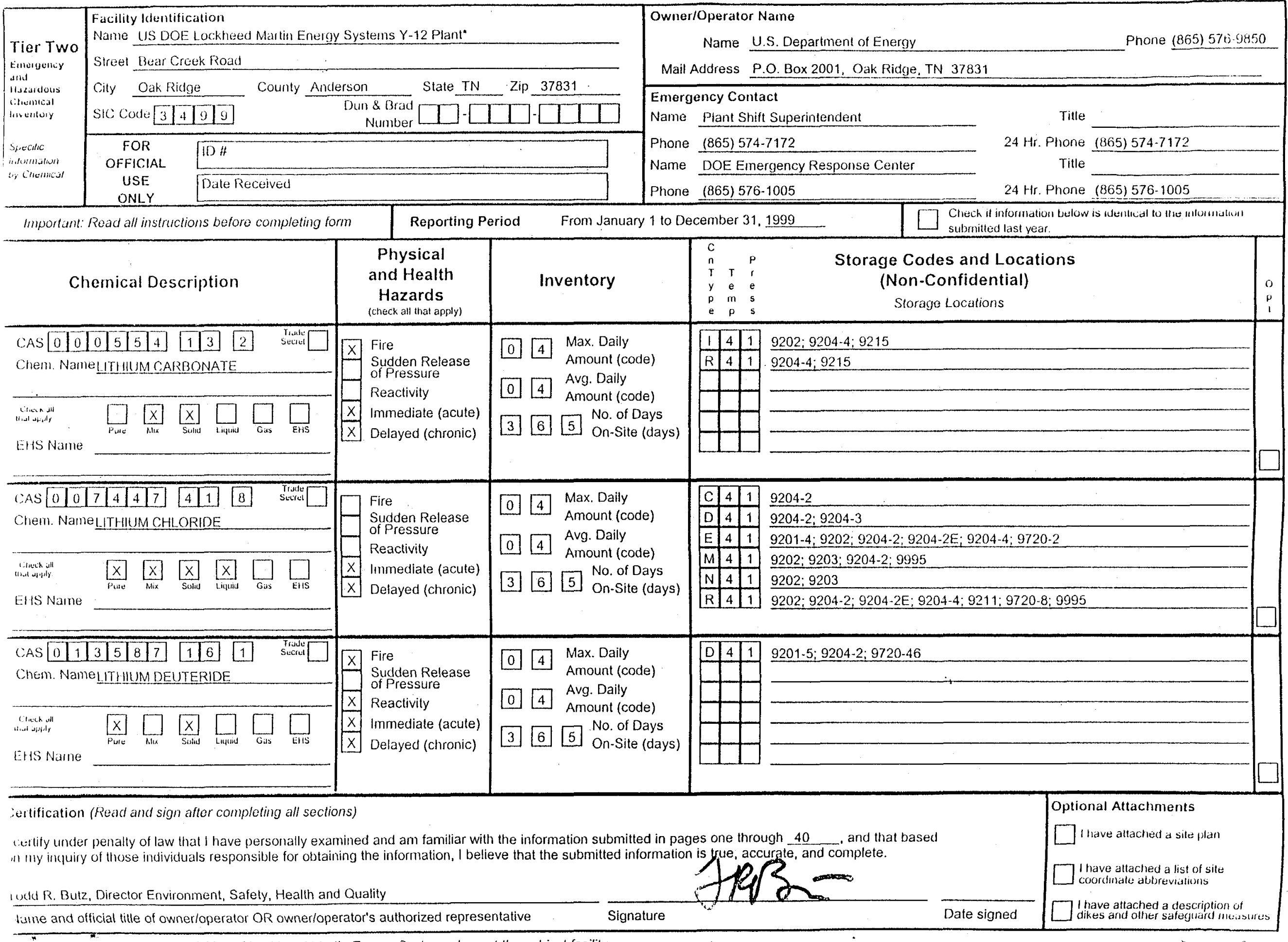

This repon covers only the activities of Lockheed Martin Energy Systems, Inc., at the subjeot facility. " 


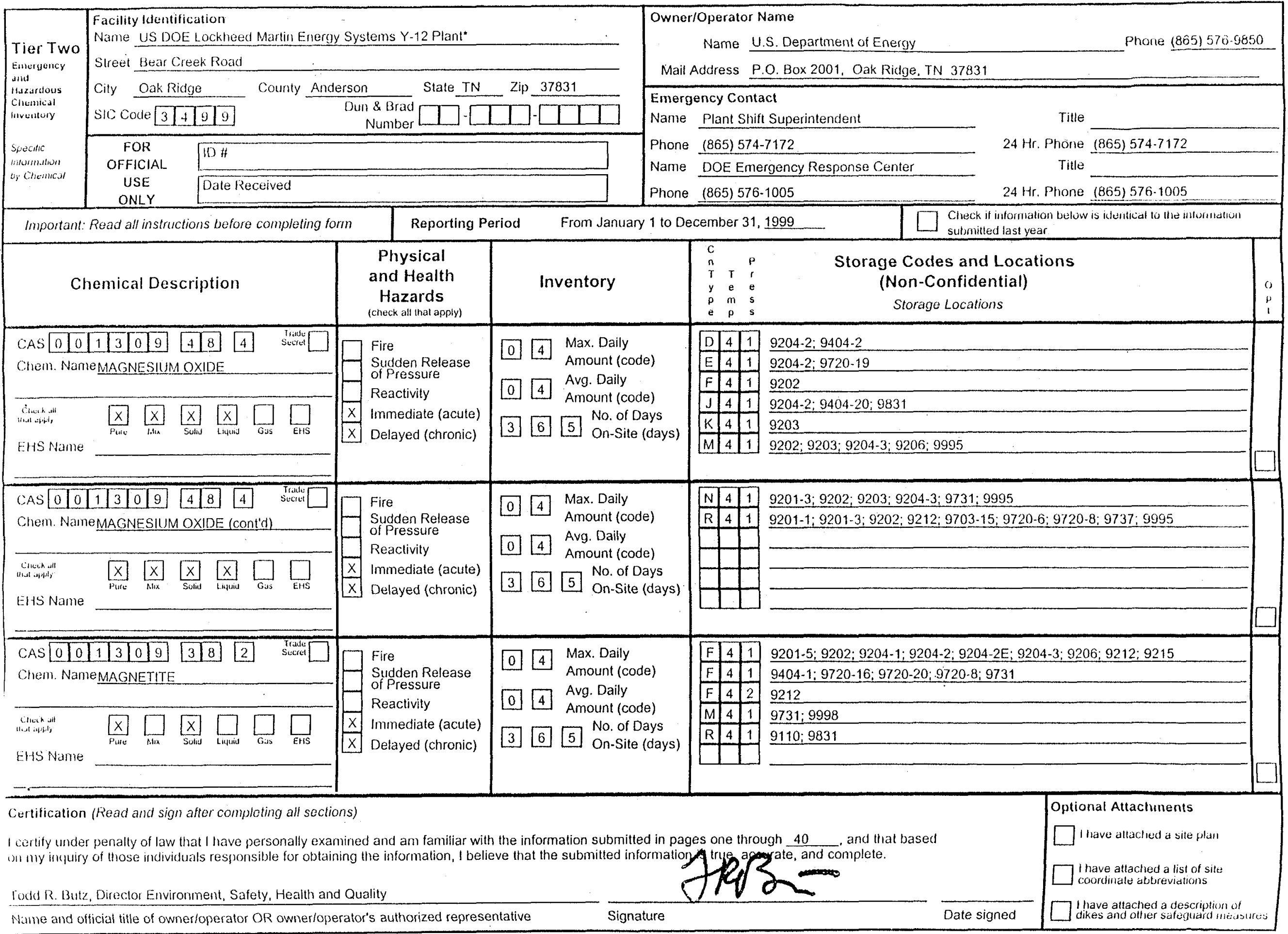

This report covers only the activitios of Lochheed Martin Energy Systems, Inc., at the subject facility. 


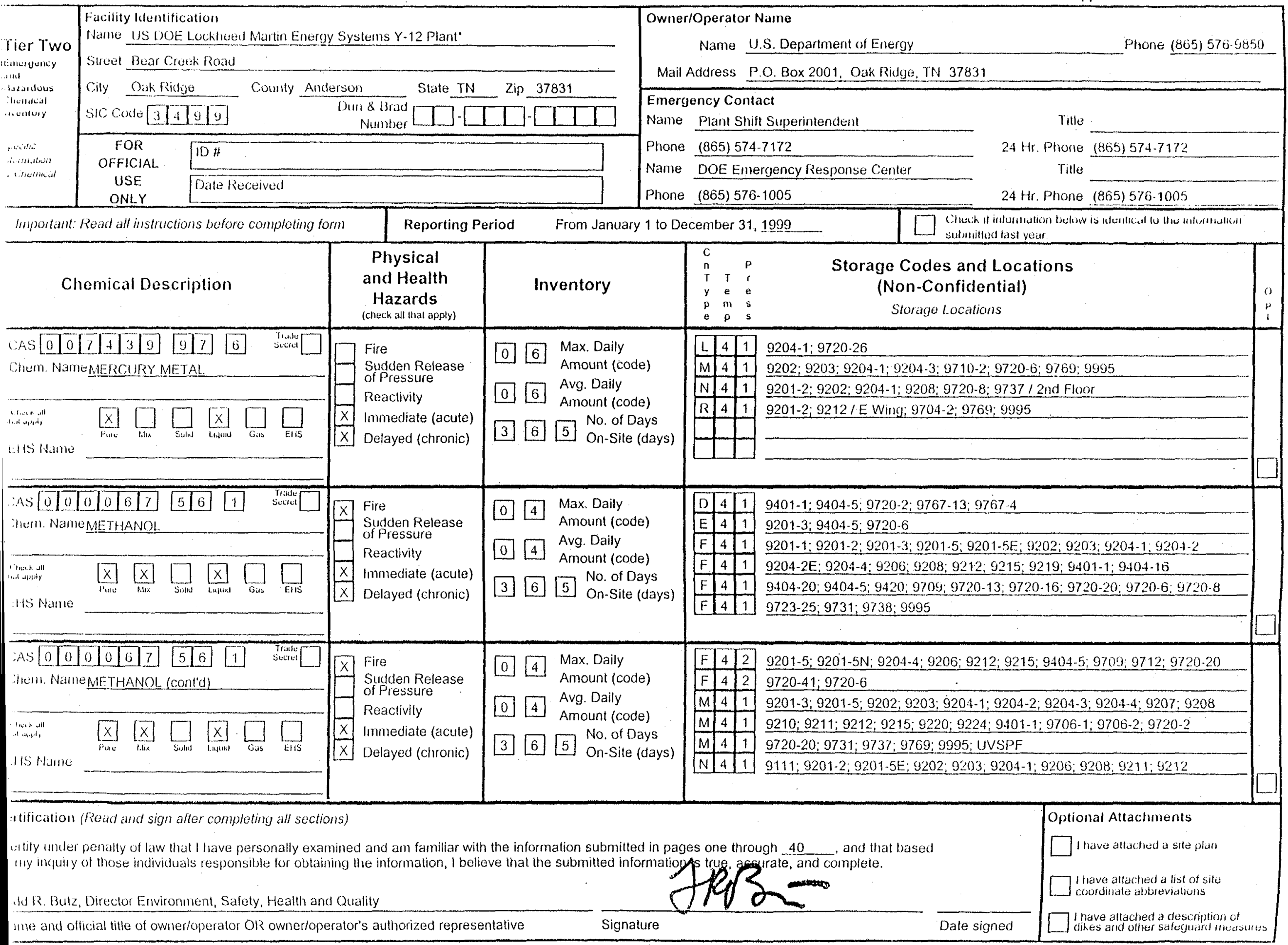

\footnotetext{
lis report covers onlly the activities of lockheed Mantin Energy Systems, Inc, at the subject facility
} 


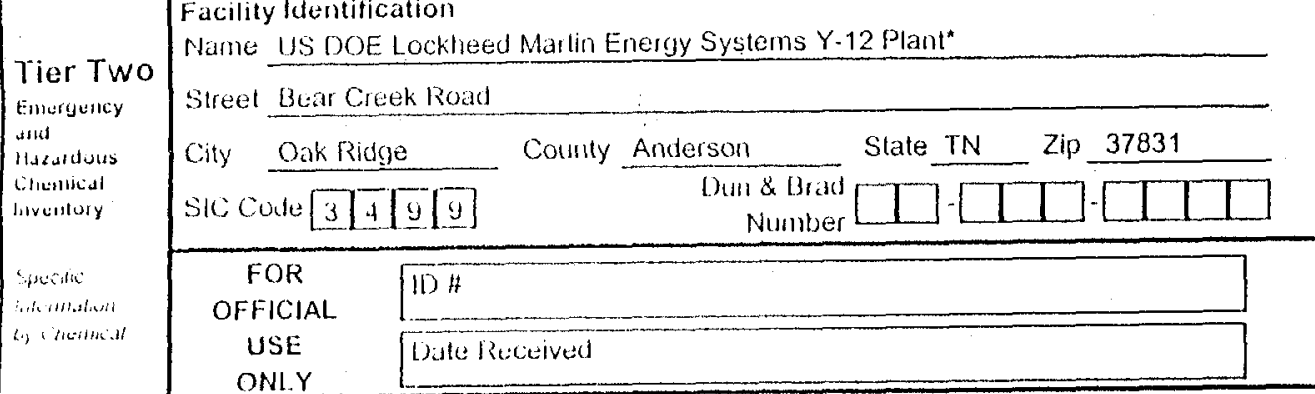

Mail Address P.O. Box 2001, Oak Ridge, TN 37831

Emergency Contact

Name Plant Shifl Superintendent

Phone (865) 574-7172

Name DOE Emergency Response Cenler

Phone (865) 576-1005

Frọm January 110 December 31, 1999

Reporting Period

Important: Read all instructions before completing form

Chenical Description

Physical

and Health

Hazards

(check all inat apply)

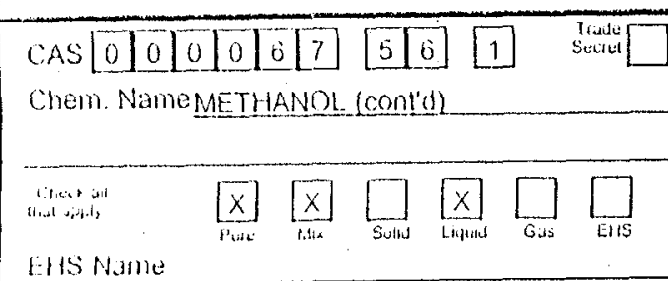

EHS Name

\section{$x$ Fire}

of Pressure

Reactivity

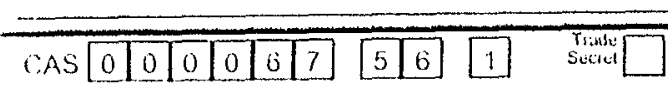

(WEm. Name METHANOL (Cont'd)

\begin{tabular}{|c|c|c|c|c|}
\hline 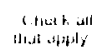 & $x$ & $x$ & $\mathrm{x}$ & $\square$ \\
\hline
\end{tabular}

EFIS Name

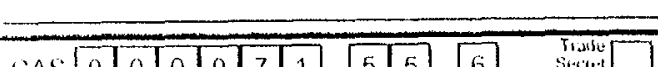

CAS \begin{tabular}{llll|l|l|l|l|l|l}
0 & 0 & 0 & 0 & 7 & 1 & 5 & 5 & 6 \\
Chem. Name METHYL CHLOROFORM
\end{tabular}

arwen ull

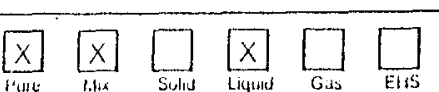

Lits Name
Inventory

$x$ Sudden Release

$x$ Immediate (acule)

$x$ Delayed (chronic)

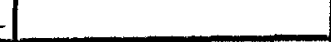

$x$ Fire

of Pressure

$\longrightarrow$ Reactivity

$x$ Immediate (acute)

$x$ Delayed (chronic) $x$

\begin{tabular}{|l|l|}
$\mathrm{x}$ & $\begin{array}{l}\text { Fire } \\
\text { Sudden Release } \\
\text { of Pressure } \\
\text { Reactivily }\end{array}$ \\
$\mathrm{x}$ & $\begin{array}{l}\text { Immediate (acule) } \\
\text { Delayed (chronic) }\end{array}$ \\
\hline
\end{tabular}

04 Max. Daily

Avg. Daily

04 Amount (code)

5] 5 No. of Days

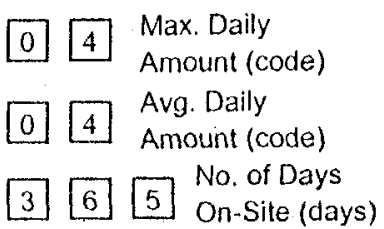

$\begin{array}{lll}C & & \\ n & & p \\ T & T & r \\ y & e & e \\ p & m & s \\ e & p & s\end{array}$

Storage Codes and Locations

(Non-Confidential)

Storage Locations

\begin{tabular}{|l|l|l|l}
\hline$N$ & 4 & 1 & $9219 ; 9401-1 ; 9401-3 ; 9404-5 ; 9420 ; 9704-1 ; 9710-3 ; 9712 ; 9714: 9720-2$
\end{tabular}

$\begin{array}{llll}\mathrm{N} & 4 & 1 & 9720-20 ; 9720-41 ; 9723-14 ; 9723-25 ; 9731 ; 9733-1 ; 9737 ; 9769 ; 9995\end{array}$

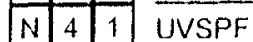

\begin{tabular}{|lll|l}
$\mathrm{N}$ & 4 & 2 & $9723-25$ \\
\hline $\mathrm{R}$ & 4 & 1 & $9103: 9201$ \\
\hline
\end{tabular}

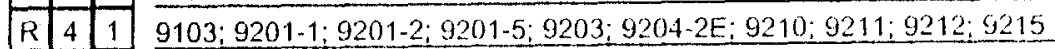

\begin{tabular}{lll|l}
\hline$R$ & 4 & 1 & $9219 ; 9220 ; 9401-1 ; 9706-2 ; 9712 ; 9714 ; 9720-2 ; 9720-6 ; 9720-8$ \\
\hline
\end{tabular}

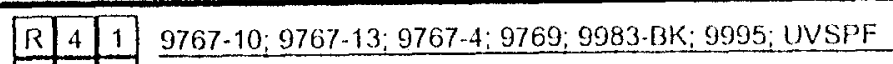

\begin{tabular}{|l|l|l|l|l|l|l|l|}
\hline$R$ & 4 & 2 & $9737 ; 9738$ \\
\hline$R$ & 6 & 2 & \\
\hline 9
\end{tabular}

\begin{tabular}{llll}
\hline$R$ & 6 & 2 & $9995 ;$ UVSPF \\
\hline
\end{tabular}

$0] 4$ Max. Daily

Amount (code)

0.7 Avg. Daily

Amount (code)

$565 \begin{aligned} & \text { No. of Days } \\ & \text { On-Site (days) }\end{aligned}$

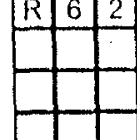

\begin{tabular}{|l|l|l|l|}
\hline$D$ & 4 & 1 \\
\hline & $9204-2 ; 9720-21$ \\
\hline
\end{tabular}

F 4 1 1 9201-1; 9201-2; 9201-3; 9201-5;9202;9203;9204-1;9204-2; $9204-4$ \begin{tabular}{llll}
\hline $\mathrm{F}$ & 4 & 1 & $9206 ; 9208 ; 9215 ; 9219 ; 9401-3 ; 9420 ; 9610 ; 9712 ; 9714 ; 9720-13$ \\
\hline
\end{tabular}

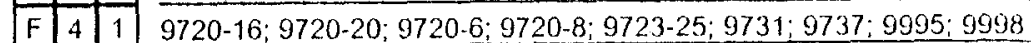

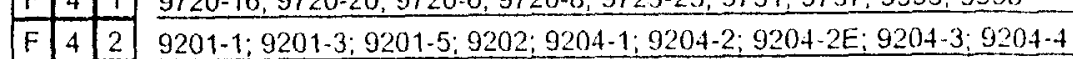
\begin{tabular}{|l|l|l|l|}
\hline$F$ & 4 & 2 \\
\hline
\end{tabular}
Curtification (Read and sign after completing all sections)

L culty under penalty of law that I have personally examined and am faniliar with the information submitted in pages one through 40 , and that based certify inder penalty of law ilhat have personally examined and am rabmitted information is true, accurate, and complete.

linfd R. Butz, Director Environment, Safety, Health and Quality

Wimte and official tille of ownerloperator OR owner/operator's authorized representative Signature

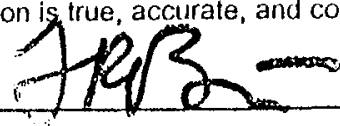

This report covers only the activities of Lochhoed Martin Energy Systoms, Inc., at the subject facility.

Optional Altachments

$\square$ irave athached à slle plan

I have attached a list of site 年 7 Ihave attached a description of Date signed 


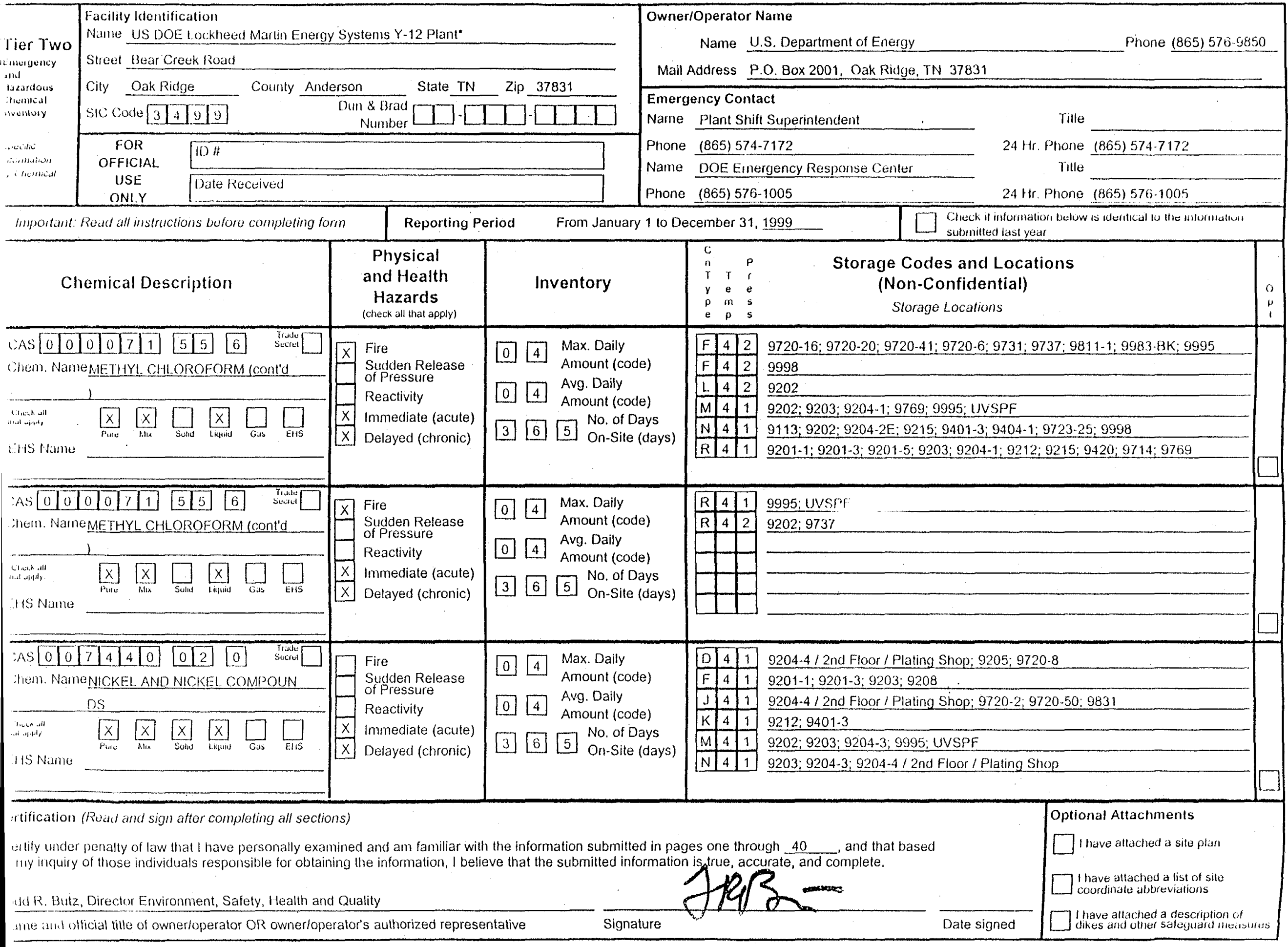

\footnotetext{
in repoit covers only the aclivities of Lockheed Martin Energy Systems, Inc., at the subject facility
} 


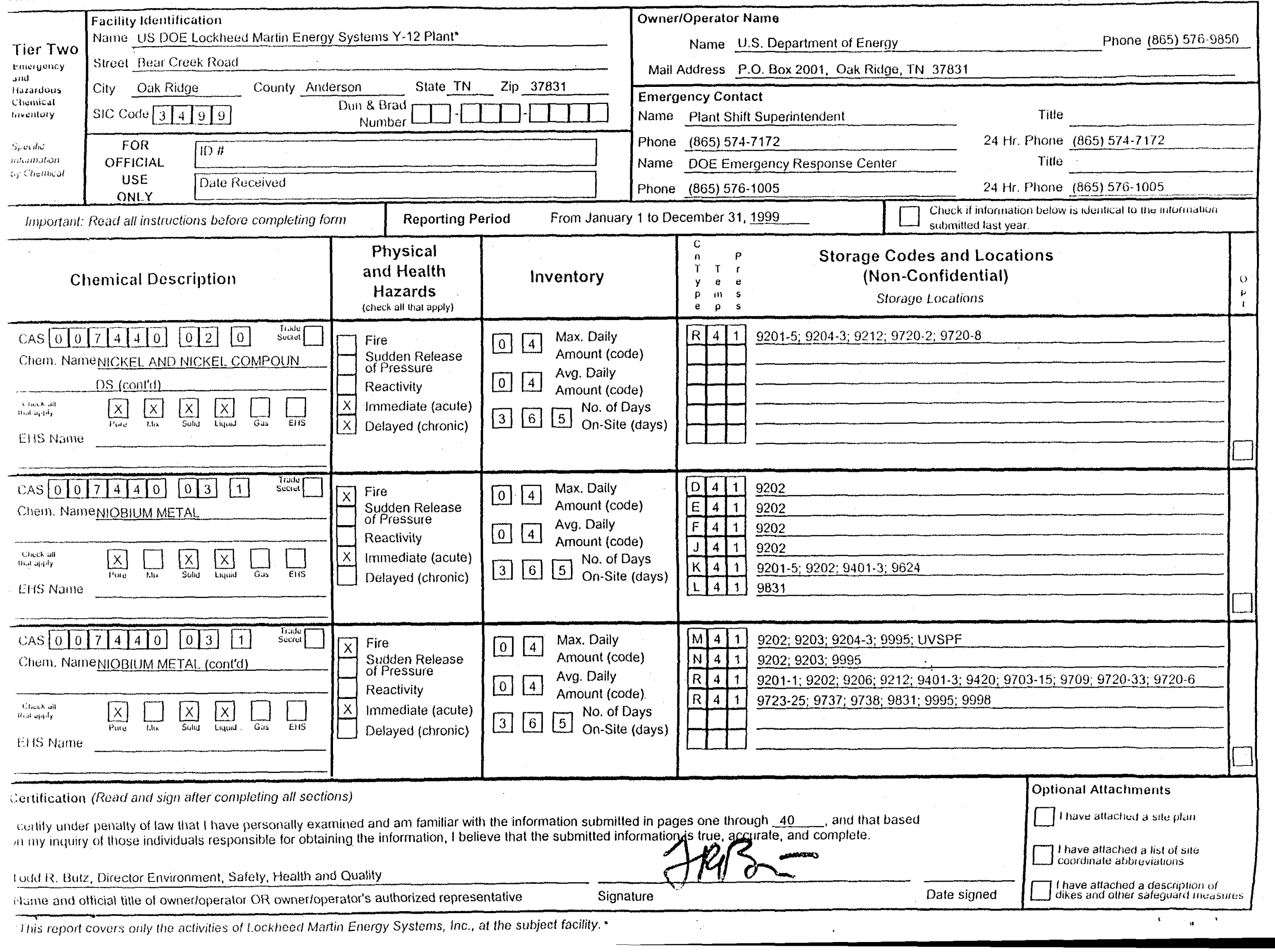




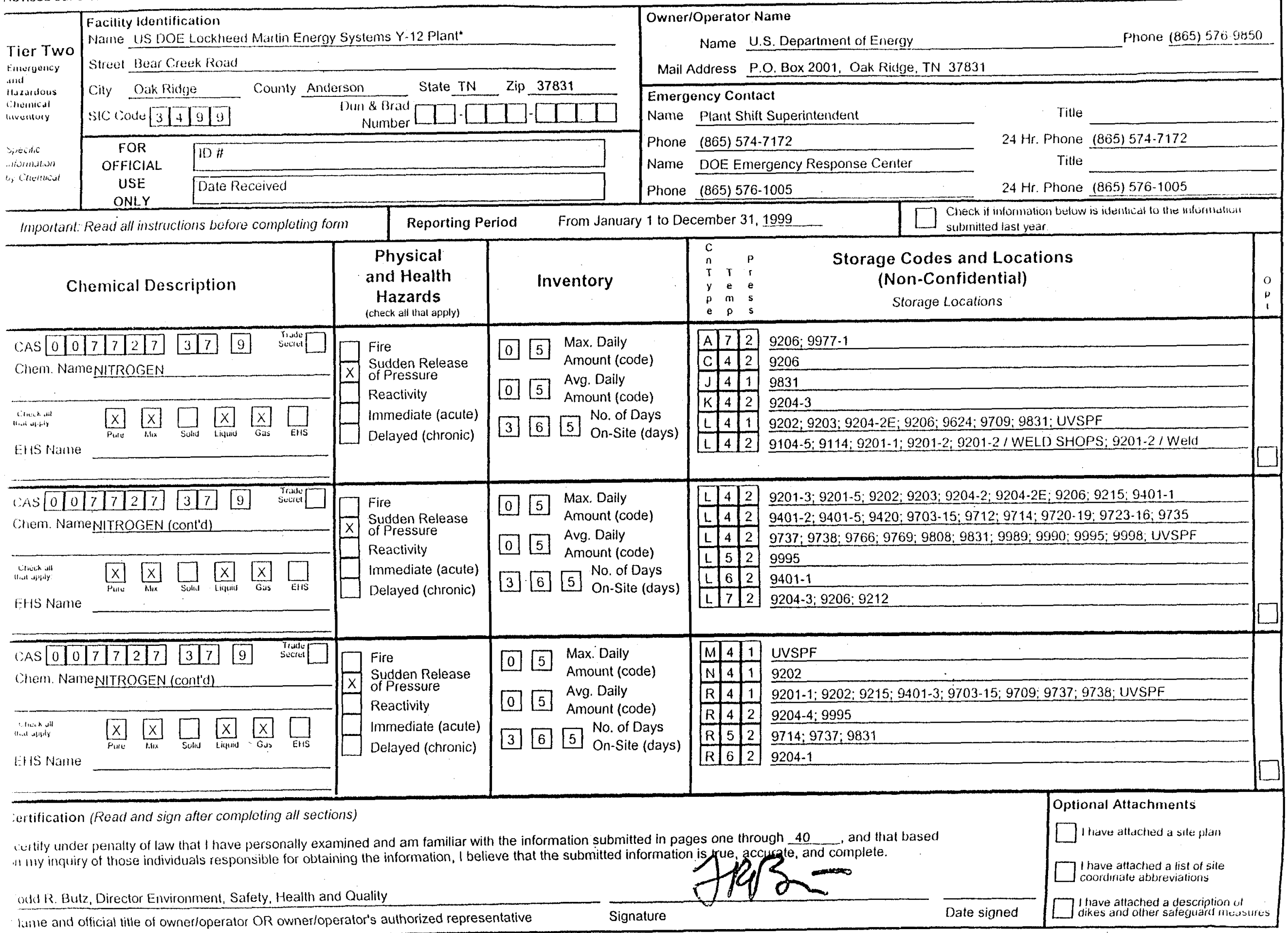

This repont covers only the activities of Lockheed Martin Energy' Systems, Inc., at the subject facility. 


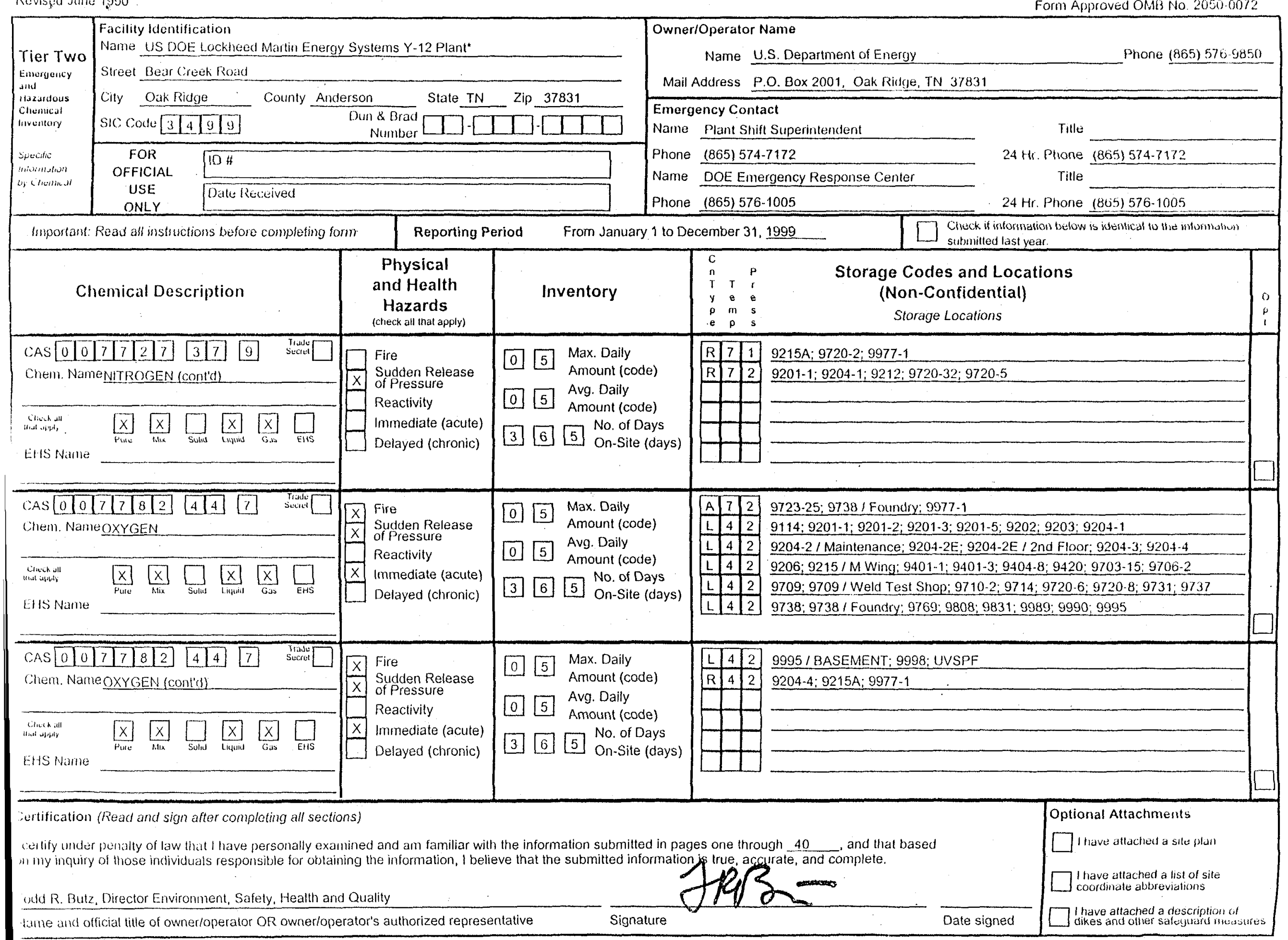

This repont covers only the activities of Lockheed Martin Energy Systems, Inc., at the subject facility. 


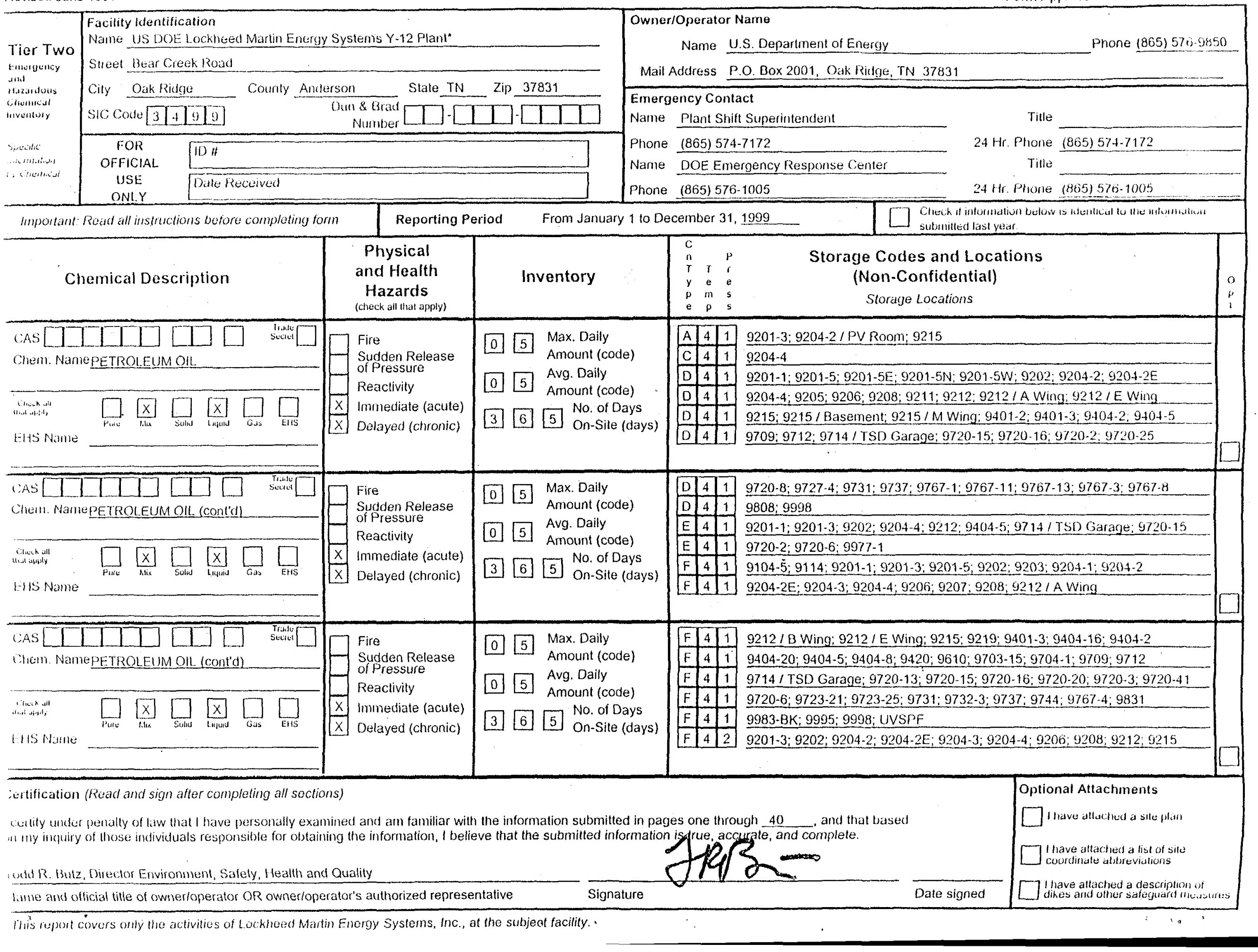






his repont covers only the activities of Lochiced Martin Energy Systems, Inc., at the subject facility. 


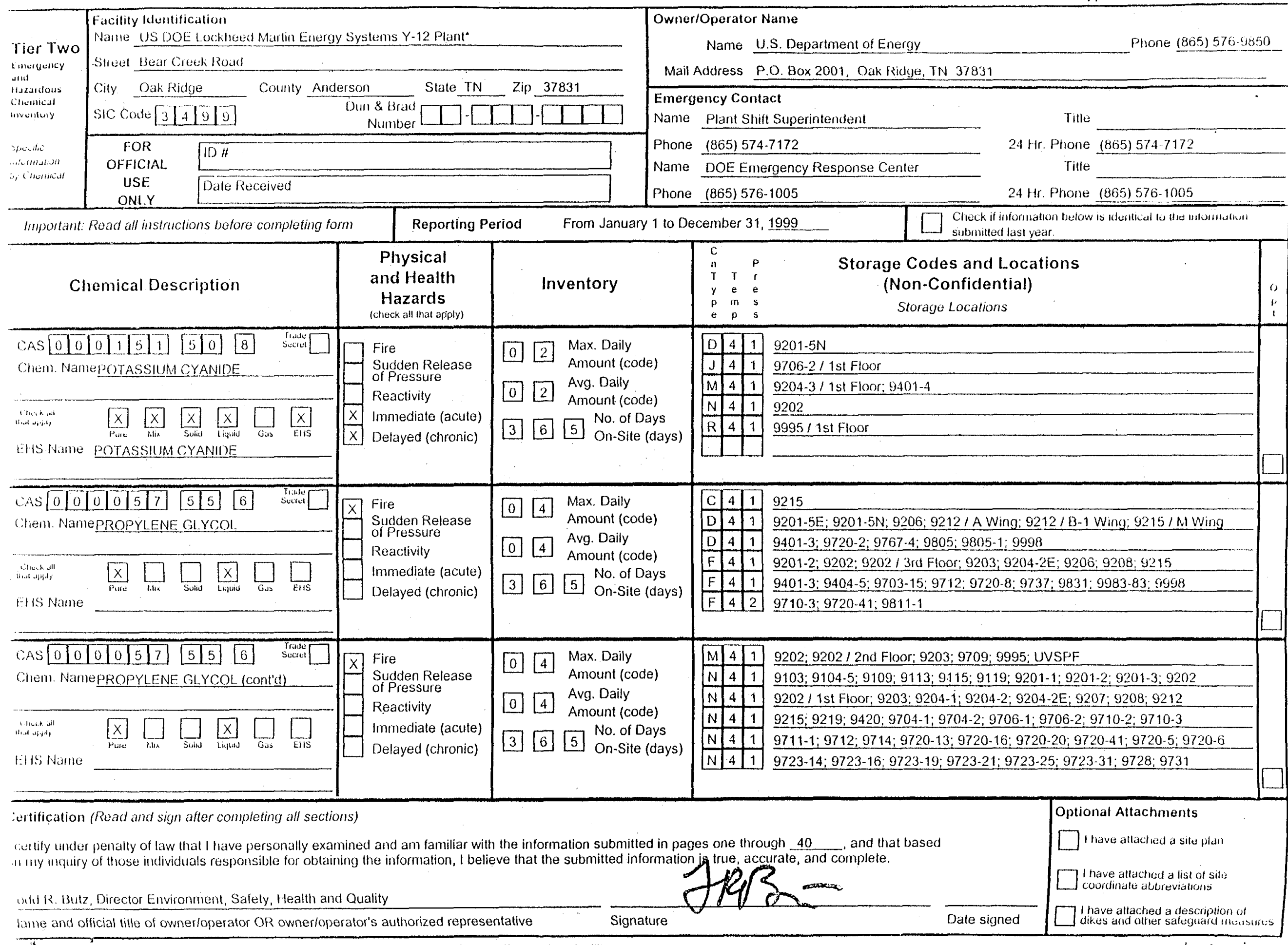

This repon covers only the activities of Lockheed Martin Energy Systems, Ine, at the subject facility. " 


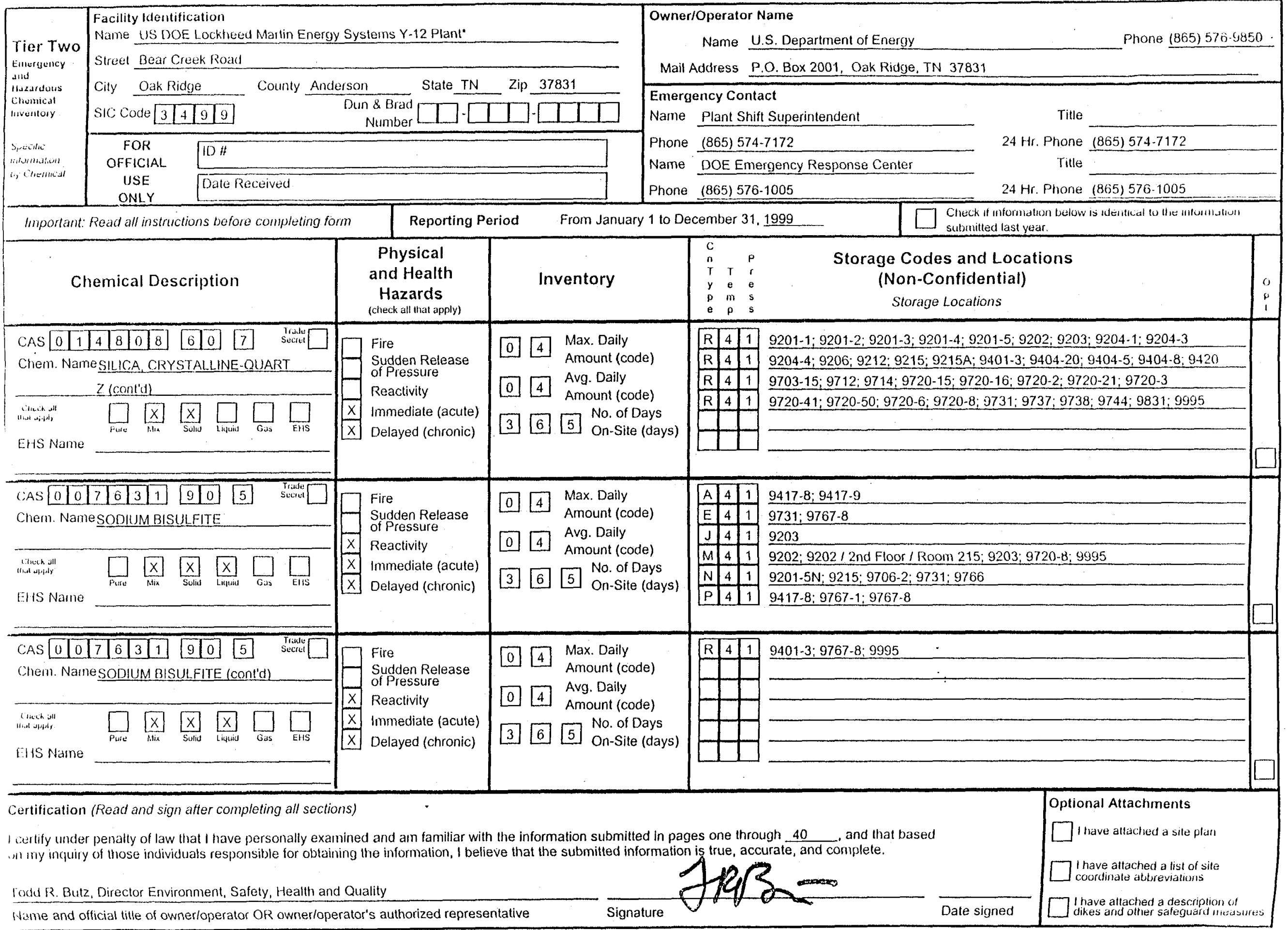

This report covers only the activities of Lockheed Martin Energy Systems, Inc., at the subject facility. 


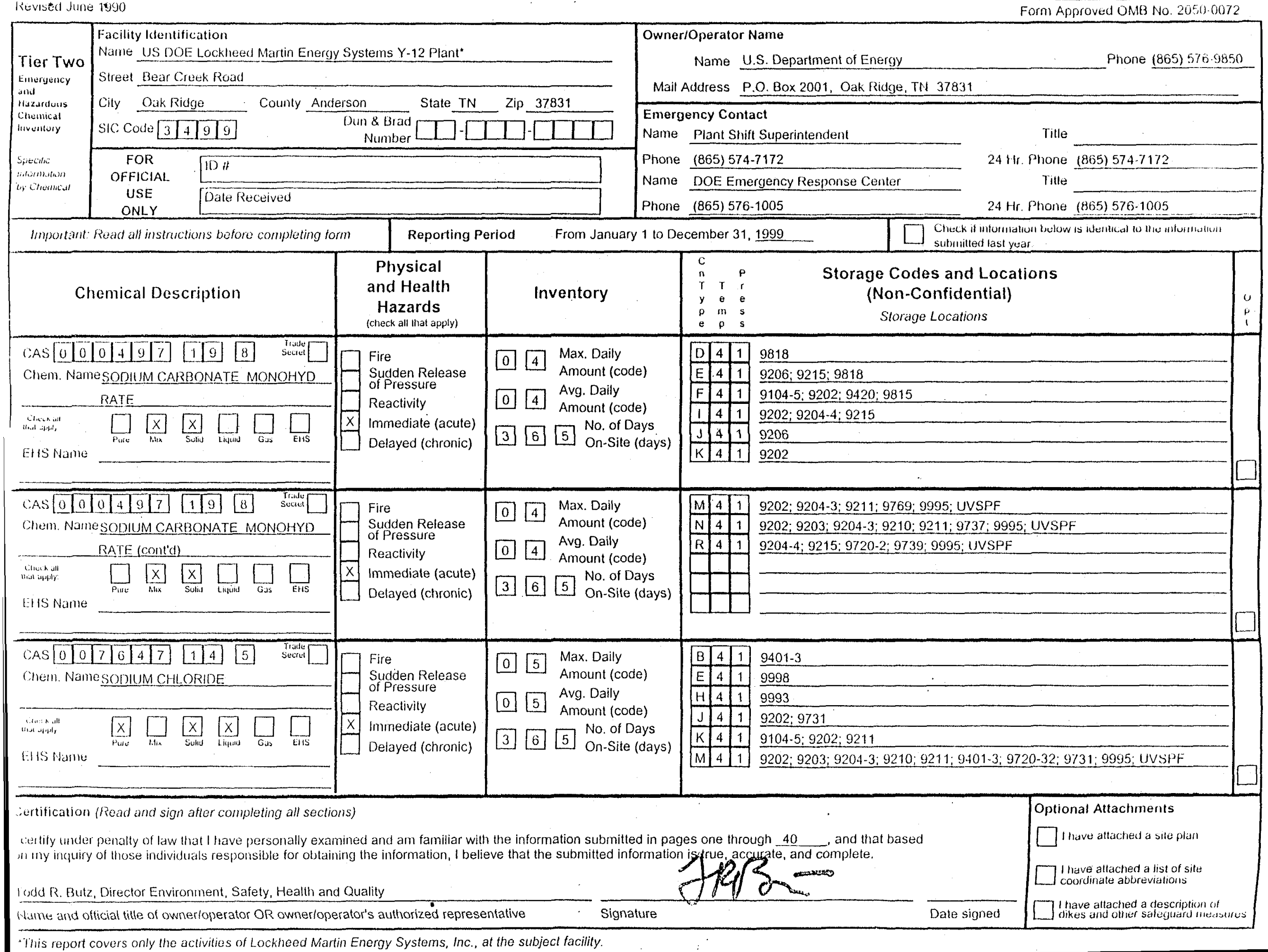

"This report covers only the activities of Lockheed Martin Energy Systems, Inc., at the subject facility. 


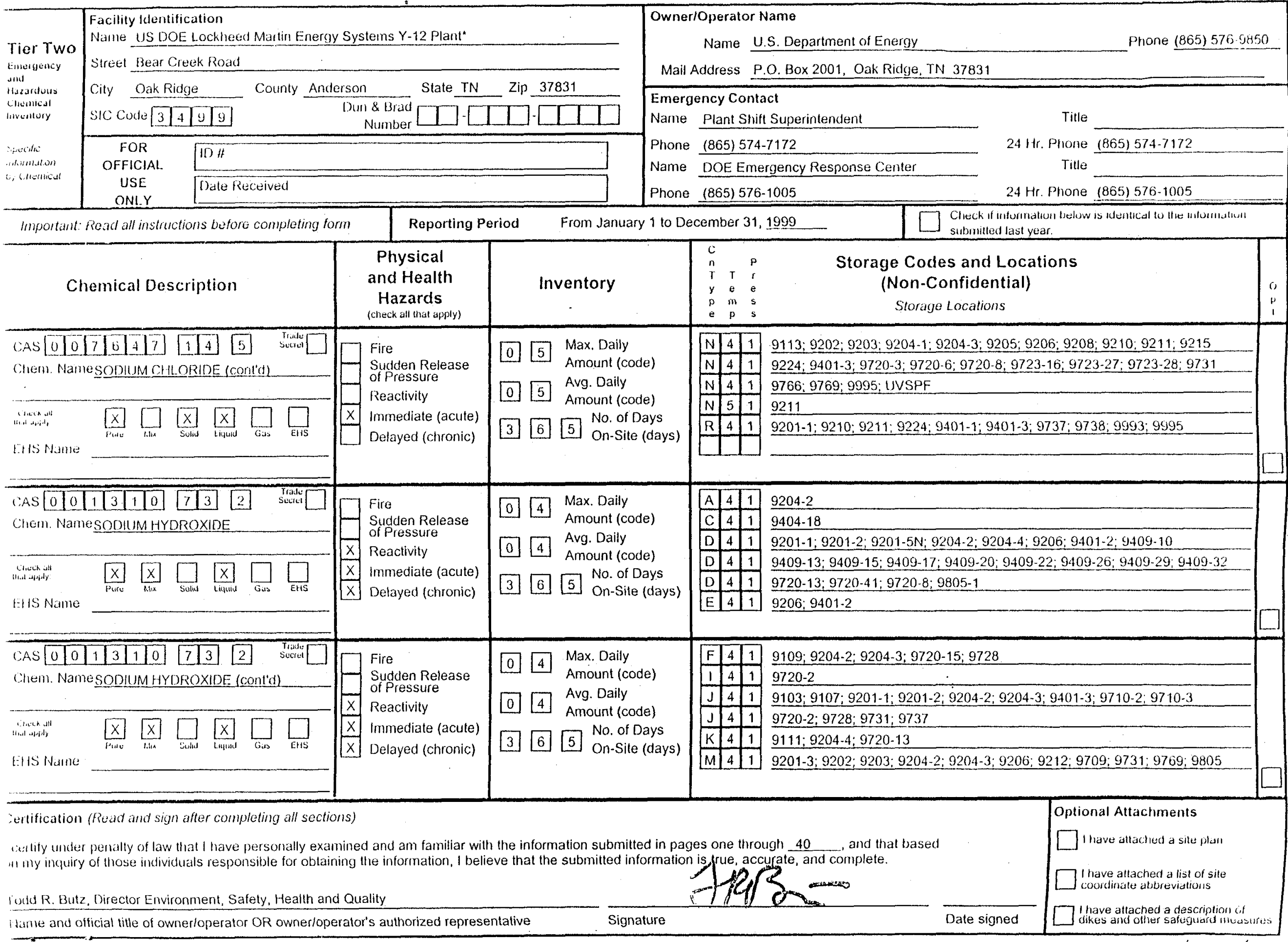

This repont covers only the activities of Lockheed Martin Energy Systems, Inc., at the subject fasility. 
lier Two

mulguicy

indisidous

theminical

intorion

- wittic

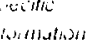

ithetintil

(thcillical

Facility Identification

Name US DOE Lockheed Martin Energy Systems Y-12 Plant*

Slleel Bear Creck Road

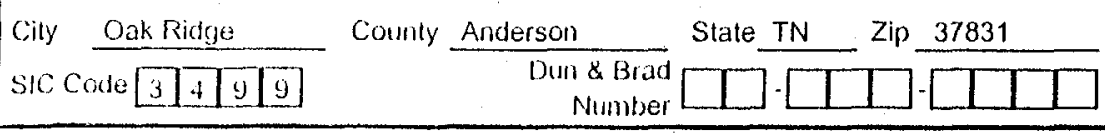

FOR

OFFICIAL

USE

ONLYY

Date Received

Non

Important Read all instructions before completing form

Chemical Description

Chemical Description

CAS[0]0[1]3[1] [7] [3]

chem. Name SODIUM HYOROXIDE (coni'd)

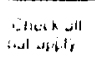

alsing

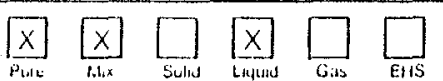

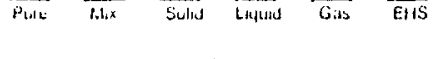

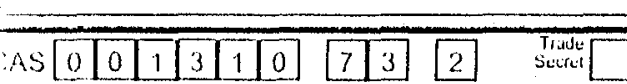

Hem. Name SOOUM HYOROXIDE (con'd)

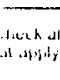

IIS Name

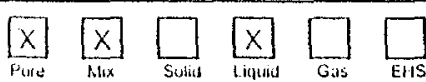

\section{Fire}

Sudden Release

$x$ Reactivity

$x$ Immediate (acule)

$x$ Delayed (chronic)

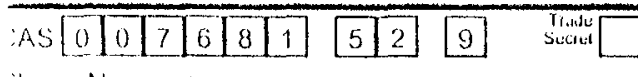

hem. NamesonIUM ryPPOCH ORTTE

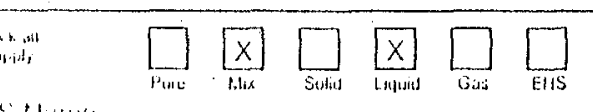

Reporting Period

\section{Physical} and Health Hazards (chieck all that apply)

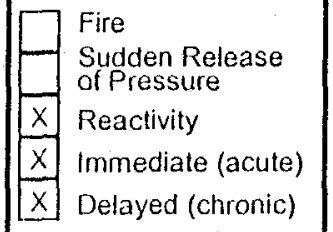

Fire

$\rightarrow$ Sucden Release

of Pressure

Reactivity

X. Immediate (acute)

Delayed (chronic)
4 Max. Daily

Amount (code)

4. Avg. Daily

Amount (code)

355 No. of Days

Phone (865) 576-1005

Name U.S. Department of Energy Phone (865) 576.0850

Mail Address P.O. Box 2001, Oak Ridge, TN 37831

\section{Emergency Contact}

Name Plant Shift Superintendent

Tille

Phone (865) 574-7172

Name DOE Emergency Response Center

1 to December 31, 1999

$-$

$\begin{array}{ccc}\mathrm{C} & & \\ n & & \mathrm{p} \\ \mathrm{T} & \mathrm{T} & \mathrm{r} \\ y & \mathrm{e} & \mathrm{e} \\ \mathrm{p} & \mathrm{m} & \mathrm{s} \\ \mathrm{e} & \mathrm{p} & \mathrm{s}\end{array}$

\begin{tabular}{|l|l|l|l|l|l|}
\hline M & 4 & 1 \\
\hline 9995; UVSPF
\end{tabular}

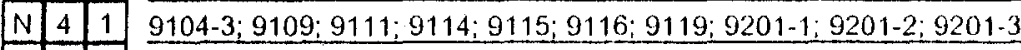

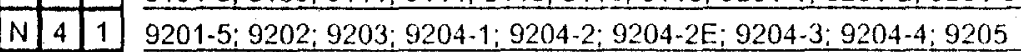

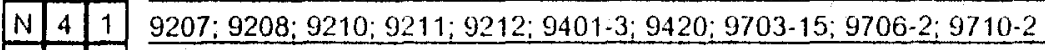

\begin{tabular}{llll}
$N$ & 4 & 1 \\
\hline & 4 & $9710-3 ; 9711-5 ; 9720-41 ; 9720-8 ; 9723-16 ; 9723-27 ; 9723-28 ; 9723-31$
\end{tabular}

\begin{tabular}{|l|l|l|}
\hline $\mathrm{N}$ & 4 & 1 \\
\hline
\end{tabular}

0] 4 Max. Daily

Amount (code)

07 Avg. Daily

Amount (code)

3 6 No. of Days

365 On-Site (days)

\begin{tabular}{|l|l|l|l|l}
\hline$R$ & 4 & 1 & $9201-1 ; 9201-2 ; 9201-3 ; 9202 ; 9204-1 ; 9204-3 ; 9204-4 ; 9207 ; 9212$
\end{tabular}

\begin{tabular}{llll}
\hline$R$ & 4 & 1 & $9401-3 ; 9404-5 ; 9409-22 ; 9720-8 ; 9737 ; 9815 ; 9995 ; 9998 ;$ UVSPF \\
\hline$R$ & 6 & 3 & $9201-2$
\end{tabular}

\begin{tabular}{|l|l|l|l|l|l|}
\hline$R$ & 6 & 3 & $9201-2$ \\
\hline
\end{tabular}

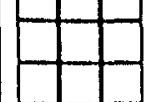

\begin{tabular}{|c|c|c|c|c|c|}
\hline$D$ & 4 & 1 \\
\hline$D$ & 4 & 1 & $9404-10 ; 9409-13 ; 9409-15 ; 9409-17 ; 9409-20 ; 9409-22 ; 9409-26 ; 9409-29$
\end{tabular}

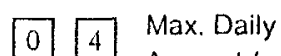

Amount (code)

Avg. Daily

4 Amount (code)

35 №. of Days

\begin{tabular}{lllll}
\hline $\mathrm{D}$ & 4 & 1 & $9409-32 ; 9720-8$ \\
\hline
\end{tabular}

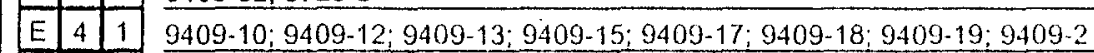

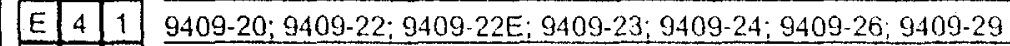

\begin{tabular}{|l|l|l|l|l}
\hline$E$ & 4 & 1 \\
\hline$M$ & 4 & 1 & $9409-32 ; 9409-34 ; 9720-2$ \\
\hline $9202 ; 9203 ; 9734$
\end{tabular}

\begin{tabular}{|l|l|l|l|l}
\hline$M$ & 4 & 1 & $9202 ; 9203: 9731$ \\
\hline
\end{tabular}

\section{rification (Read and sign after completing all sections)}$$
\text { 1! }
$$

y under penalty of law that I have personally examined and am familiar with the information submitted in pages one through 40 and that based

incuuiry of those individials responsible for obtaining the information, I believe that the submitted information isfrue, accurate, and complete.

AR. Butz, Director Environment, Safety, Health and Quality

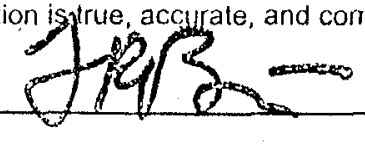

Optional Attachments

ne and official title of owner/operator OR owner/operator's authorized representative

Signature

Date signed

$\square$ inlave attachlied a sile plan

I have attached a list of site coordinale abureviatuoris I have attached a description of
dikets and other saleguard incistuic

Ieport covers only the activitics of Lockheed Martin Energy Systems, Inc., at the subject facility. 


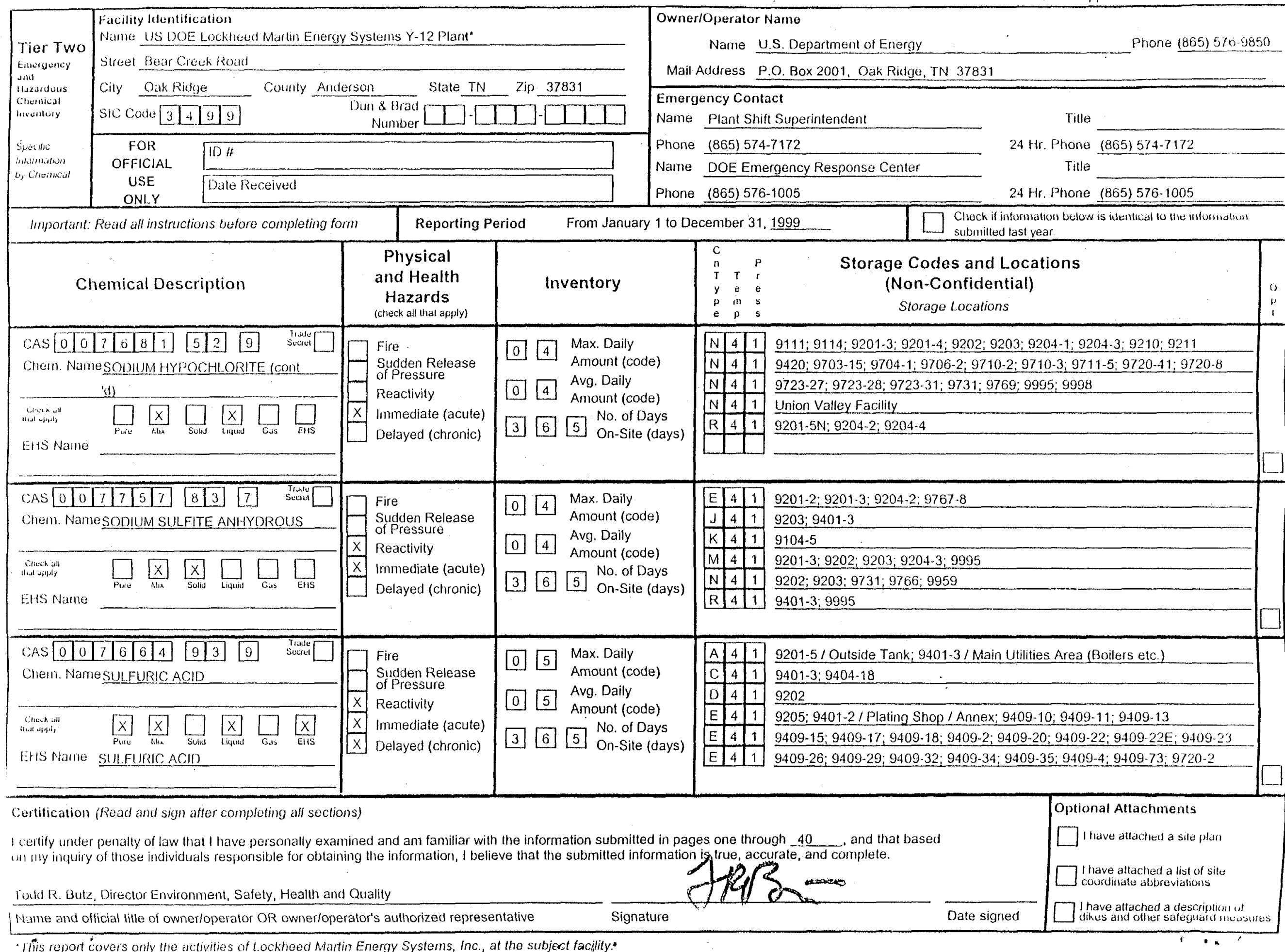




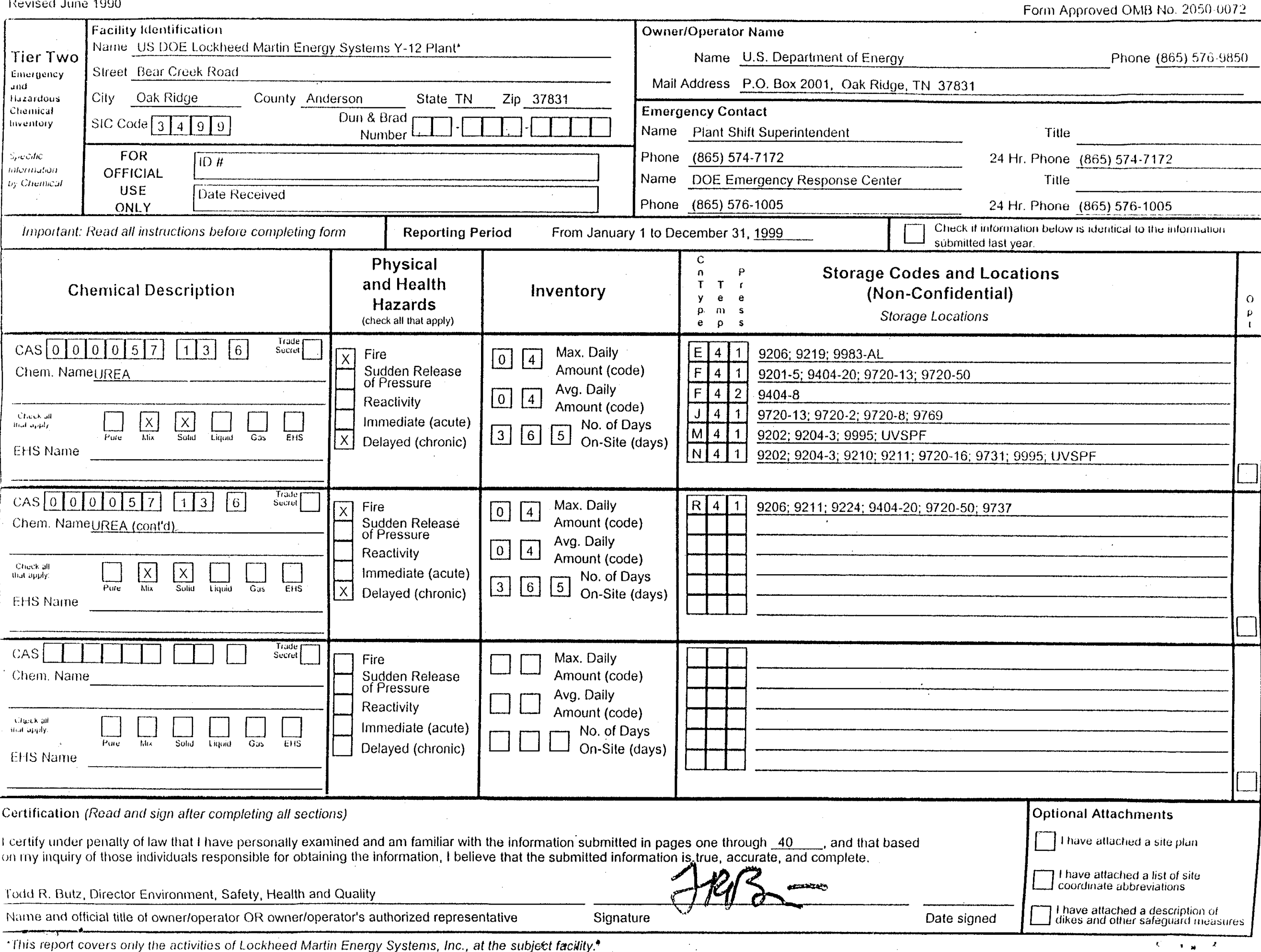


Distribution:

J. D. Bolling

J. M. Eaton/R. A. Evans

R. T. Ford

M. Gallahar, DOE-EOC

H. S. Hackler

J. J. Hummer, LMC

E. Irwin

M. E. Lemmings, DOE-ORO

R. W. Martin, DOE-ORO

J. D. Rothrock/B. E. Cochran, DOE-ORO

R. R. Smith

M. Vestal, TDEC

Y-12 Records Services (1)/DOE-OSTI (3)

Y-12 Plant Shift Superintendent

EC Document Center-RC 